\title{
Nonlinear hierarchical control of a quad tilt-wing UAV: An adaptive control approach
}

\author{
Yildiray Yildiz $^{1}$ | Mustafa Unel $^{2}$ | Ahmet Eren Demirel ${ }^{2}$
}

${ }^{1}$ Department of Mechanical Engineering, Bilkent University, Cankaya, Ankara 06800, Turkey

${ }^{2}$ Faculty of Engineering and Natural Sciences, Sabanci University, Tuzla, Istanbul 34956, Turkey

\section{Correspondence}

Mustafa Unel, Faculty of Engineering and Natural Sciences, Sabanci University, Tuzla, Istanbul 34956, Turkey.

Email: munel@sabanciuniv.edu

\begin{abstract}
Summary
In this paper, a nonlinear hierarchical adaptive control framework is proposed for the control of a quad tilt-wing unmanned aerial vehicle (UAV). An outer loop model reference adaptive controller with robustifying terms creates required forces to be able to move the UAV on a reference trajectory, and an inner loop nonlinear adaptive controller realizes the required attitude angles to achieve these forces. A rigorous stability analysis is provided showing the boundedness of all the signals in this cascaded controller structure. The development and the stability analysis of the controller do not use any linearizations and use the full nonlinear UAV dynamics. The controller is implemented on a high-fidelity nonlinear tilt-wing quadrotor model in the presence of uncertainties, wind disturbances, and measurement noise as well as actuator and structural failures. In this work, in addition to earlier modeling studies, the effect of wing-angle variations, actuator failures, and structural failures and their effect on the center of gravity of the UAV are rigorously and systematically investigated and reflected in the model. Simulation results showing the performance of the proposed controller and a comparison with the fixed controller used in earlier studies are presented in the paper.
\end{abstract}

\section{KEYWORDS}

adaptive control, hierarchical control, MRAC, tilt-wing UAV, VTOL

\section{1 | INTRODUCTION}

Significant progress has been made in the design, modeling, and control of unmanned aerial vehicles (UAVs) in the last decade, and much recent work has been devoted to the investigation of hybrid-wing UAVs. Hybrid-wing UAVs are platforms where the advantages of rotary-wing and fixed-wing UAVs are combined: They can achieve vertical takeoff and landing (VTOL) without the assistance of any special infrastructure, and they can fly for extended periods with high speed. A subclass under the hybrid-wing UAVs is tilt-rotor UAVs, which constitute the characteristic of efficient energy use. $^{1,2}$ In this subclass, one can find dual tilt-rotor ${ }^{3}$ and dual tilt-wing UAVs. ${ }^{4}$ Quad-tilt wing UAVs ${ }^{5,6}$ form another category, which do not show the disadvantage of cyclic control requirement that can be seen in its dual tilt-rotor counterparts.

Nomenclature: $m$, mass, $\mathrm{kg} ; I_{b}$, inertia matrix in the body frame, $\mathrm{kg} \cdot \mathrm{m}^{2} ; V_{w}$, linear velocity in the world frame, $\mathrm{m} / \mathrm{s} ; \Omega_{b}$, angular velocity in the body frame, $\mathrm{rad} / \mathrm{s} ; \Omega_{w}$, angular velocity in the world frame, $\mathrm{rad} / \mathrm{s} ; F_{t}$, net force, $\mathrm{N} ; M_{t}$, net moment, $\mathrm{N} \cdot \mathrm{m} ; X, Y, Z$, coordinates of the center of mass in the world frame, $\mathrm{m} ; p, q, r$, angular velocities in the body frame, $\mathrm{rad} / \mathrm{s} ; \Phi, \Theta, \Psi$, roll, pitch and yaw angles in the world frame, rad; $\omega_{i}$, rotational speed of the $i^{\text {th }}$ rotor, $\mathrm{rad} / \mathrm{s} ; M$, inertia matrix; $\zeta$, vector defined as $[X, Y, Z, p, q, r]^{T} ; \xi$, vector defined as $[X, Y, Z, \Phi, \Theta, \Psi]^{T} ; C(\zeta)$, Coriolis-centripetal matrix; $G$, gravity vector, $\mathrm{m} \cdot \mathrm{s}^{-2} ; I_{x x}, I_{y y}, I_{z z}$, moments of inertia around the body axes, $\mathrm{kg} \cdot \mathrm{m}^{2} ; O\left(\zeta, \omega_{i}\right)$, gyroscopic term; $E\left(\xi, \omega_{i}^{2}\right)$, actuator vector; $\theta_{f}$, front wing angle, rad; $k$, motor thrust constant, $\mathrm{N} \cdot \mathrm{s}^{2} \cdot \mathrm{rad}^{-2} ; l_{s}$, rotor distance to center of mass along y axis, $\mathrm{m} ; l_{l}$, rotor distance to center of mass along x axis, m; $\lambda$, torque/force ratio; $W(\xi)$, wing force vector, $\mathrm{N} ; R_{b w}$, rotation matrix representing the orientation of the body frame w.r.t. world frame; $F_{D}, F_{L}$, drag and lift forces, $\mathrm{N}$; $v_{x}, v_{y}, v_{z}$, linear velocities in the body frame, $\mathrm{m} / \mathrm{s} ; \dot{X}, \dot{Y}, Z$, linear velocities in the world frame, $\mathrm{m} / \mathrm{s} ; v_{\alpha}$, variable defined as $\sqrt{v_{x}^{2}+v_{z}^{2}} ; \theta_{i}$, wing angle of attack w.r.t. body $\mathrm{x}$ axis, rad; $\alpha_{i}$, effective angle of attack of the wing w.r.t. air flow, $\operatorname{rad} ; \rho$, air density, $\mathrm{kg} \cdot \mathrm{m}^{-3} ; A$, wing planform area, $\mathrm{m}^{2} ; R\left(\theta_{i}-\alpha_{i}\right), \operatorname{rotation}$ matrix around body y axis; $\alpha_{w}$, vector defined as $[\Phi, \Theta, \Psi]^{T} ; E\left(\alpha_{w}\right)$, velocity transformation matrix between from the world frame to the body frame. 
The couplings between the translational and rotary motions, highly nonlinear multi-input multioutput system dynamics, uncertainty sources such as unpredictable damages, and actuator malfunctions ${ }^{7}$ are challenges that make the control of tilt-wing UAVs a difficult task, which requires advanced controllers if high performance throughout a large flight envelope is demanded. There exists a rich literature on the closed-loop control of rotary UAVs offering a variety of controllers to handle these challenges. Some examples of controllers proposed in the literature are proportional-integral-derivative (PID)-type controllers, ${ }^{8-11}$ PD2 controllers where a proportional and 2 derivative actions are used, ${ }^{12}$ linear quadratic regulator controllers, ${ }^{9,13-15}$ sliding mode observers with feedback linearization, ${ }^{16} H_{\infty}$ controllers, ${ }^{17}$ feedback linearization approaches, ${ }^{18}$ nonlinear model predictive control, ${ }^{19}$ dynamic inversion with $\mu$ synthesis, ${ }^{20}$ nested nonlinear controllers, ${ }^{21}$ backstepping approaches, ${ }^{22-24}$ and some other nonlinear control techniques. ${ }^{25}$ There are other control methods proposed in the literature that use a hierarchical structure ${ }^{26-30}$ with various types of controllers for rotational and translational dynamics. An excellent comprehensive literature survey about the guidance, navigation, and control of rotary UAVs can be found in 1 study. ${ }^{31}$

All the above-mentioned control approaches proved successful in simulation and experimental environments for specific operating conditions. The proposed controller in this paper is built upon these earlier works by eliminating the precise plant model requirement for the optimization-based and classical approaches and by eliminating the conservatism introduced by the robust control approaches. This is achieved by using a hierarchical nonlinear adaptive controller where adaptive controllers are used both for the translational and rotational motion control and thus providing adaptation in all 6 degrees of freedom, together with a rigorous stability analysis for the overall cascaded closed-loop system. There exist other adaptive control approaches in the literature for the control of rotary UAVs such as the ones proposed in previous studies ${ }^{32-35}$ and a very recent one in the work of Dydek et al. ${ }^{7}$ However, the hierarchical adaptive control framework proposed in this paper is different from them: Unlike in the work of Johnson and Kannan, ${ }^{32}$ the proposed control framework does not use any neural networks and therefore computationally less expensive; unlike this study, ${ }^{33}$ no small angle or slowly varying parameter assumptions are made; unlike another study, ${ }^{34}$ no fuzzy approximators are used and again, computationally cheaper; unlike another study, ${ }^{35}$ uncertainties in both the translational and rotational motion are addressed, and finally, unlike this study, ${ }^{7}$ no linearization is conducted on plant dynamics. In addition, none of these mentioned adaptive control approaches are implemented on a tilt-wing UAV.

It is known that the problem of nonlinear controllers, in general, is computational complexity. ${ }^{36}$ The focus of this paper is the design of a high-performance, practical controller that is easy to implement with low computational cost but at the same time theoretically sound that does not need any linearization approximation of the plant dynamics and that has a rigorous overall stability proof. In addition, the proposed controller is able to compensate the uncertainties in plant dynamics, due to online wing-angle variations, damages, and unexpected component failures. All these features are realized by using 2 adaptive controllers in cascade: A model reference adaptive control (MRAC) design for the translational dynamics and a nonlinear adaptive controller for the rotational dynamics. Although these approaches are well known, their cascaded implementation in a hierarchical framework for the hybrid-wing UAVs has not been shown to result in a stable closed-loop system before, to the best of authors' knowledge. The proof of stability of this overall closed-loop system is achieved by the help of lumping the inner loop errors as a disturbance term and using robustifying terms in the adaptive controller design. Therefore, the implementation of 2 computationally cheap adaptive controllers in a cascaded form together with a stability proof provides the well-needed control framework, which is practical, adaptive, and theoretically sound at the same time.

The proposed control framework is implemented for the control of a novel quad tilt-wing UAV (SUAVI: Sabanci University Unmanned Aerial Vehicle), shown in Figure 1. The characteristics that make SUAVI a novel quad tilt-wing UAV and its advantages over existing designs can be found in 1 study. ${ }^{37}$ Sabanci University Unmanned Aerial Vehicle was previously designed, manufactured, and flight tested by Unel et al, and earlier research results have been published about

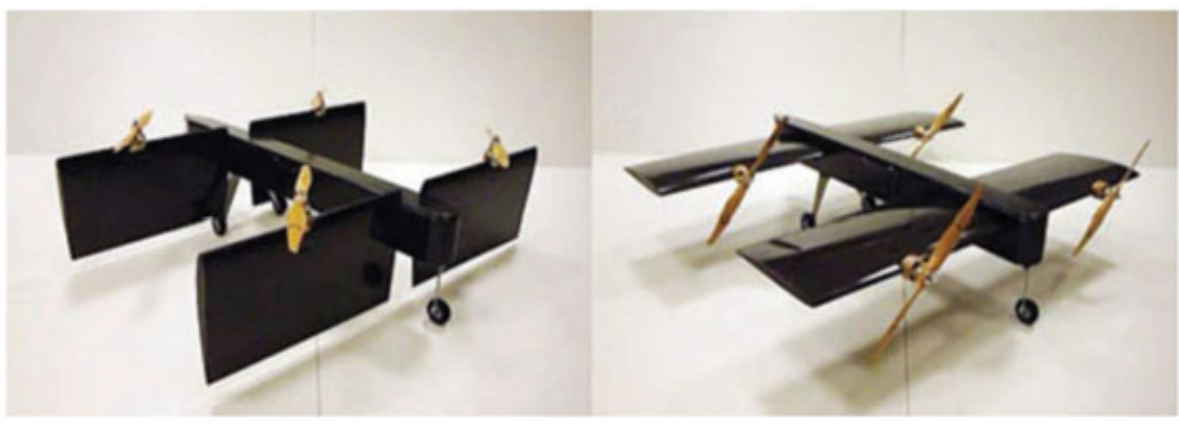

FIGURE 1 Quad tilt-wing unmanned aerial vehicle (Sabanci University Unmanned Aerial Vehicle) with 2 different wing-angle configurations. [Colour figure can be viewed at wileyonlinelibrary.com] 
the aerodynamic and mechanical design, prototyping, control system design, and flight tests. ${ }^{37-40}$ However, in those studies, the effects of wing-angle variations, component failures, and unexpected damages on system uncertainty were not investigated and the controller was not tested against these adverse conditions. The designed controller was a fixed controller without any adaptation capability and thus had limited capabilities to compensate for uncertainties. In addition, although showed promising results in flight tests, the overall closed-loop control system did not have a rigorous stability proof and relied on the time-scale separation between the inner and outer loop controllers. In this paper, SUAVI is tested, via a high-fidelity simulation model, which was used during the development of the actual prototype, for the above-mentioned adverse conditions using a theoretically sound controller. Especially, the effect of wing-angle variations on system dynamics is rigorously analyzed and the uncertainty it produces when combined with possible asymmetry between the wings and probable failures is quantified. A systematic study of wing-angle variations for tilt-wing UAVs and its effect on the adaptive closed loop control system was not rigorously investigated before. This is especially important during the transition phase between the vertical and the horizontal motions where the wings move from a vertical position (quadrotor behavior, Figure 1, left picture) to almost horizontal position (fixed-wing behavior, Figure 1, right picture). This investigation also proposes a method of transition from the quadrotor mode to fixed-wing mode. In addition, energy conservation, compared to conventional quadrotors, with the help of lift creation with wings during horizontal flight is quantified.

To summarize, the contributions of this study are the following. On the theory side, a novel nonlinear hierarchical adaptive controller is proposed where (1) each controller is computationally cheap, (2) both the overall hierarchical framework and individual controllers are easy to implement, (3) no linearization is needed in plant dynamics, and (4) overall closed-loop stability proof is provided. To the best of authors' knowledge, no such combination of adaptive controllers for a hierarchical control framework is used in earlier studies, especially with a rigorous stability proof. On the practical side, the proposed controller is implemented on a high-fidelity model of a novel quad tilt-wing UAV developed by the authors, where (1) uncertainties emanating from a combination of wing asymmetry, component failure, and unexpected damages are quantified, (2) the effect of wing-angle variation during the transition phase on plant dynamics is quantified in a systematic manner, and (3) an approach is proposed for transition between the quadrotor phase (vertical wing) and the fixed-wing phase (almost horizontal wing). No earlier study exists about the adaptive control of quad tilt-wing UAVs that considers all these phenomena at the same time.

Preliminary simulation results of this study are presented in 1 study ${ }^{41}$ Different from the work of Yildiz et al, ${ }^{41}$ in this study, a rigorous stability analysis, implementation results with disturbances and noise, and a rigorous quantification of uncertainties due to wing movements and failures are presented.

The organization of the paper is as follows: System model is presented in Section 2. Controller design and stability investigation are presented in Section 3. Implementation scenario together with uncertainty quantification and trajectory generation is presented in Section 4 . Simulation results are presented in Section 5, and a summary is given in Section 6.

\section{2 | SYSTEM MODEL}

Equations of motion for the quad tilt-wing UAV are briefly presented in this section. A more detailed analysis can be found in 1 study. ${ }^{37}$ Overall dynamic equations of the system are given as

$$
\left[\begin{array}{cc}
m I_{3 \times 3} & 0_{3 \times 3} \\
0_{3 \times 3} & I_{b}
\end{array}\right]\left[\begin{array}{c}
\dot{V}_{w} \\
\dot{\Omega}_{b}
\end{array}\right]+\left[\begin{array}{c}
0 \\
\Omega_{b} \times\left(I_{b} \Omega_{b}\right)
\end{array}\right]=\left[\begin{array}{c}
F_{t} \\
M_{t}
\end{array}\right],
$$

where $m$ and $I_{b}$ represent the mass and the diagonal inertia matrix in the body frame and $V_{w}$ and $\Omega_{b}$ represent the linear and the angular velocities of the vehicle in the world and body frames, respectively. The net force and the moment applied on the vehicle are represented by $F_{t}$ and $M_{t}$, respectively (see Figure 2). It should be noted that for tilt-wing quadrotors, these forces and moments are functions of the rotor trusts and wing angles.

Using vector-matrix notation, Equation 1 can be rewritten as follows:

$$
M \dot{\zeta}+C(\zeta) \zeta=G+O\left(\zeta, \omega_{i}\right)+E\left(\xi, \omega_{i}^{2}\right)+W(\xi),
$$

where,

$$
\zeta=[\dot{X}, \dot{Y}, Z, p, q, r]^{T},
$$

and

$$
\xi=[X, Y, Z, \Phi, \Theta, \Psi]^{T},
$$

where $X, Y$, and $Z$ are the coordinates of the center of mass with respect to the world frame, $p, q$, and $r$ are the angular velocities in the body frame, $\Phi, \Theta$, and $\Psi$ are the roll, pitch, and yaw angles of the vehicle expressed in the world frame, and $\omega_{i}, i=1,2,3,4$ represents the rotor rotational speeds. $M$, the inertia matrix, $C$, Coriolis-centripetal matrix, and $G$, the gravity term, are given as follows:

$$
M=\left[\begin{array}{cc}
m I_{3 \times 3} & 0_{3 \times 3} \\
0_{3 \times 3} & \operatorname{diag}\left(I_{x x}, I_{y y}, I_{z z}\right)
\end{array}\right],
$$




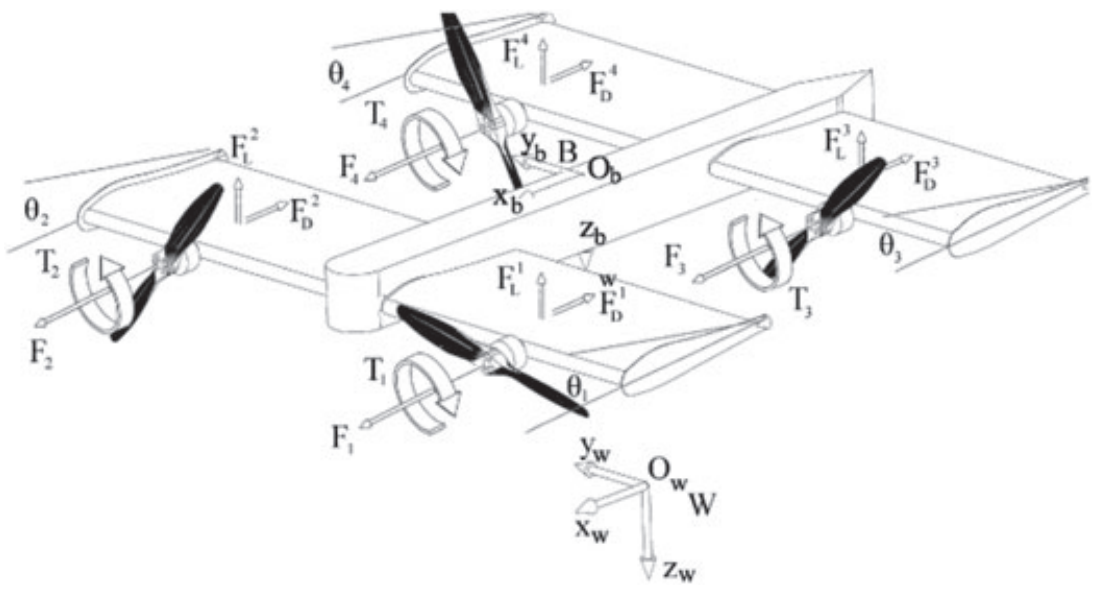

FIGURE 2 Forces and moments on the quad tilt-wing unmanned aerial vehicle

$$
\begin{gathered}
C(\zeta)=\left[\begin{array}{cccccc}
0 & 0 & 0 & 0 & 0 & 0 \\
0 & 0 & 0 & 0 & 0 & 0 \\
0 & 0 & 0 & 0 & 0 & 0 \\
0 & 0 & 0 & 0 & I_{z z} r & -I_{y y} q \\
0 & 0 & 0 & -I_{z z} r & 0 & I_{x x} p \\
0 & 0 & 0 & I_{y y} q & -I_{x x} p & 0
\end{array}\right], \\
G=[0,0, m g, 0,0,0]^{T},
\end{gathered}
$$

where, $I_{x x}, I_{y y}$, and $I_{z z}$ are the moments of inertia around the body axes. The gyroscopic term, $O(\zeta, \omega)$, is given as

$$
O\left(\zeta, \omega_{i}\right)=J_{\text {prop }}\left(\sum_{i=1}^{4}\left[\eta_{i} \Omega_{b} \times\left[\begin{array}{c}
0_{\theta_{i}} \\
0 \\
-s_{\theta_{i}}
\end{array}\right] \omega_{i}\right),\right.
$$

where, $\eta_{(1,2,3,4)}=1,-1,-1,1$ and $c_{\theta_{i}}$ and $s_{\theta_{i}}$ represent cosine and sine of the wing angles, respectively. When 2 simplifying assumptions are used, namely, neglecting the aerodynamic downwash effect of the front wings on the rear wings and using same angles for the front and rear wings, system actuator vector, $E\left(\xi, \omega^{2}\right)$, can be given as

$$
E\left(\xi, \omega_{i}^{2}\right)=\left[\begin{array}{c}
\left(c_{\Psi} c_{\Theta} c_{\theta_{f}}-\left(c_{\Phi} s_{\Theta} c_{\Psi}+s_{\Phi} s_{\Psi}\right) s_{\theta_{f}}\right) u_{1} \\
\left(s \Psi c_{\Theta} c_{\theta_{f}}-\left(c_{\Phi} s_{\Theta} S_{\Psi}-s_{\Phi} c_{\Psi}\right) s_{\theta_{f}}\right) u_{1} \\
\left(-s_{\Theta} c_{\theta_{f}}-c_{\Phi} c_{\Theta} s_{\theta_{f}}\right) u_{1} \\
s_{\theta_{f}} u_{2}-c_{\theta_{f}} u_{4} \\
s_{\theta_{f}} u_{3} \\
c_{\theta_{f}} u_{2}+s_{\theta_{f}} u_{4}
\end{array}\right],
$$

where $\theta_{f}$ represents front wing angle against the UAV body $\mathrm{X}$-axis. Inputs $u_{1}, u_{2}, u_{3}$, and $u_{4}$ in Equation 9 are given as

$$
\begin{aligned}
& u_{1}=k\left(\omega_{1}^{2}+\omega_{2}^{2}+\omega_{3}^{2}+\omega_{4}^{2}\right), \\
& u_{2}=k l_{s}\left(\omega_{1}^{2}-\omega_{2}^{2}+\omega_{3}^{2}-\omega_{4}^{2}\right), \\
& u_{3}=k l_{l}\left(\omega_{1}^{2}+\omega_{2}^{2}-\omega_{3}^{2}-\omega_{4}^{2}\right), \\
& u_{4}=k \lambda\left(\omega_{1}^{2}-\omega_{2}^{2}-\omega_{3}^{2}+\omega_{4}^{2}\right),
\end{aligned}
$$

where $k, l_{s}, l_{l}$, and $\lambda$ are the motor thrust constant, rotor distance to center of mass along $\mathrm{y}$-axis, rotor distance to center of mass along $\mathrm{x}$-axis, and torque/force ratio, respectively.

The wing forces $W(\xi)$, lift and drag, and the moments they create on the UAV are given as

$$
W(\zeta)=\left[\begin{array}{c}
R_{b w}\left[\begin{array}{c}
F_{D}^{1}+F_{D}^{2}+F_{D}^{3}+F_{D}^{4} \\
0 \\
F_{L}^{1}+F_{L}^{2}+F_{L}^{3}+F_{L}^{4}
\end{array}\right] \\
0 \\
l_{l}\left(F_{L}^{1}+F_{L}^{2}-F_{L}^{3}-F_{L}^{4}\right) \\
0
\end{array}\right],
$$

where $R_{b w}$ is the rotation matrix representing the orientation of the body frame with respect to world frame. The drag and lift forces, $F_{D}^{i}=F_{D}^{i}\left(\theta_{f}, v_{x}, v_{z}\right)$ and $F_{L}^{i}=F_{L}^{i}\left(\theta_{f}, v_{x}, v_{z}\right)$, are given as

$$
\left[\begin{array}{c}
F_{D}^{i} \\
0 \\
F_{L}^{i}
\end{array}\right]=R\left(\theta_{i}-\alpha_{i}\right)\left[\begin{array}{c}
-\frac{1}{2} c_{D}\left(\alpha_{i}\right) \rho A v_{\alpha}^{2} \\
0 \\
-\frac{1}{2} c_{L}\left(\alpha_{i}\right) \rho A v_{\alpha}^{2}
\end{array}\right],
$$

where $v_{\alpha}=\sqrt{v_{x}^{2}+v_{z}^{2}}$ and $\alpha_{i}=\theta_{i}-\left(-\operatorname{atan} 2\left(v_{z}, v_{x}\right)\right)$. Here, $\rho$ is the air density, $A$ is the wing planform area and $\alpha_{i}$ is the effective angle of attack of the wing with respect to the air flow, and $\theta_{i}$ is the wing angle of attack with respect to the body $\mathrm{x}$-axis. $R\left(\theta_{i}-\alpha_{i}\right)$ is the rotation matrix around $\mathrm{y}$-axis that decomposes the forces on the wings on the body axis.

The relationship between the linear velocities in body frame $v_{x}, v_{y}, v_{z}$ and linear velocities in the world frame $\dot{X}, \dot{Y}, Z$ is given as

$$
\left[\begin{array}{c}
v_{x} \\
v_{y} \\
v_{z}
\end{array}\right]=R_{w b}(\Phi, \Theta, \Psi)\left[\begin{array}{c}
\dot{X} \\
\dot{Y} \\
Z
\end{array}\right] .
$$

Using Equation 1, the following rotational dynamics, that is, in a form suitable for attitude controller design, is obtained:

$$
M\left(\alpha_{w}\right) \dot{\Omega}_{w}+C\left(\alpha_{w}, \Omega_{w}\right) \Omega_{w}=E^{T} M_{t},
$$


where $\alpha_{w}=[\Phi, \Theta, \Psi]^{T}, \Omega_{w}=[\dot{\Phi}, \dot{\Theta}, \dot{\Psi}]$ and $E\left(\alpha_{w}\right)$ is the velocity transformation matrix, which is given as

$$
E\left(\alpha_{w}\right)=\left[\begin{array}{ccc}
1 & 0 & -s_{\Theta} \\
0 & c_{\Phi} & s_{\Phi} c_{\Theta} \\
0 & -s_{\Phi} & c_{\Phi} c_{\Theta}
\end{array}\right] .
$$

The relationship between the angular velocity of the UAV in the body frame, $\Omega_{b}$, and in the world frame, $\Omega_{w}$, is given as

$$
\Omega_{b}=\left[\begin{array}{c}
p \\
q \\
r
\end{array}\right]=E\left(\alpha_{w}\right) \Omega_{w} .
$$

The contents of the modified inertia matrix $M\left(\alpha_{w}\right)$ in Equation 17 and their derivation can be found in previous study. ${ }^{41}$

\section{3 | CONTROLLER DESIGN}

To control the position of the tilt-wing UAV, whose nonlinear dynamics was provided in Section 2, we used a hierarchical nonlinear control approach that can adapt its parameters online. On the upper level, an MRAC is designed that provides virtual control inputs to control the position of the UAV. These control inputs are converted to desired attitude angles, which are then fed to the lower-level attitude controller. A nonlinear adaptive controller is designed as the attitude controller so that uncertainties can be compensated without the need for linearization of system dynamics. Closed-loop control system structure is presented in Figure 3, and upper and lower level controllers are described below.

\section{1 | MRAC design}

An MRAC, that resides in the upper level of the hierarchy, is designed to control the position of the vehicle, assuming that the system is a simple mass. This controller calculates the required forces that need to be created, by the lower-level nonlinear controller, in the $X, Y$, and $Z$ directions, to make the UAV follow the desired trajectory. No information is used about the actual mass of the UAV during the design, and this uncertainty in the mass is handled by online modification of control parameters based on the trajectory error. It is noted that the uncertainties in moment of inertia are handled by the lower-level attitude controller, which is explained in the next section.

Consider the following system dynamics, which is obtained using the translational part of Equation 2 and assuming that it is possible to implement control forces parallel to the $\mathrm{x}$-axis, $\mathrm{y}$-axis, and $\mathrm{z}$-axis of the world frame:

$$
\begin{aligned}
\dot{X}(t) & =A \underline{X}(t)+B_{n} \Lambda\left(u_{M R A C}(t)+D+W_{l d}+\pi(t)\right), \\
y(t) & =C \underline{X}(t),
\end{aligned}
$$

where $\underline{X}=[X, Y, Z, \dot{X}, \dot{Y}, X]^{T} \in \Re^{6}$ is the state vector, $u_{M R A C} \in \mathfrak{R}^{3}$ is the position controller signal (see Figure 3), $W_{l d} \equiv\left[W_{x} W_{y} W_{z}\right]^{T} \in \mathfrak{R}^{3}$ is the lift and drag forces, which are given in the first 3 rows of Equation $14, \pi(t) \in \Re^{3}$ is a bounded, time-varying, unknown disturbance, and $y \in \mathfrak{R}^{3}$ is the plant output.

$$
\begin{gathered}
A=\left[\begin{array}{ll}
0_{3 \times 3} & I_{3 \times 3} \\
0_{3 \times 3} & 0_{3 \times 3}
\end{array}\right], \\
B_{n}=\left[\begin{array}{c}
0_{3 \times 3} \\
I_{3 \times 3}
\end{array}\right] \frac{1}{m_{n}}, \\
\Lambda=\frac{m_{n}}{m}, \\
D=\left[\begin{array}{c}
0_{2 \times 1} \\
m g
\end{array}\right], \\
C=\left[\begin{array}{ll}
I_{3 \times 3} & 0_{3 \times 3}
\end{array}\right],
\end{gathered}
$$

where $m$ is the actual mass of the UAV that is assumed to be unknown, $m_{n}$ is the nominal mass, $g$ is the gravitational acceleration, and $\Lambda$ represents the uncertainty in the UAV mass. It is noted that from now on, time dependence of the parameters will not be emphasized unless necessary and therefore " $t$ " will be dropped from the expressions.

Remark 1. The model introduced in Equation 20 represents a simple mass being controlled via virtual control inputs acting in the direction of 3 axes of the world frame in the presence of gravity, lift and drag forces, and unknown and bounded time-varying disturbances. It is noted that this representation would be accurate if the inner loop controller, which controls the attitude of the UAV, had infinite bandwidth, which is not the case. The errors due to the dynamics of the inner loop controller and their effects on the boundedness of the solutions of

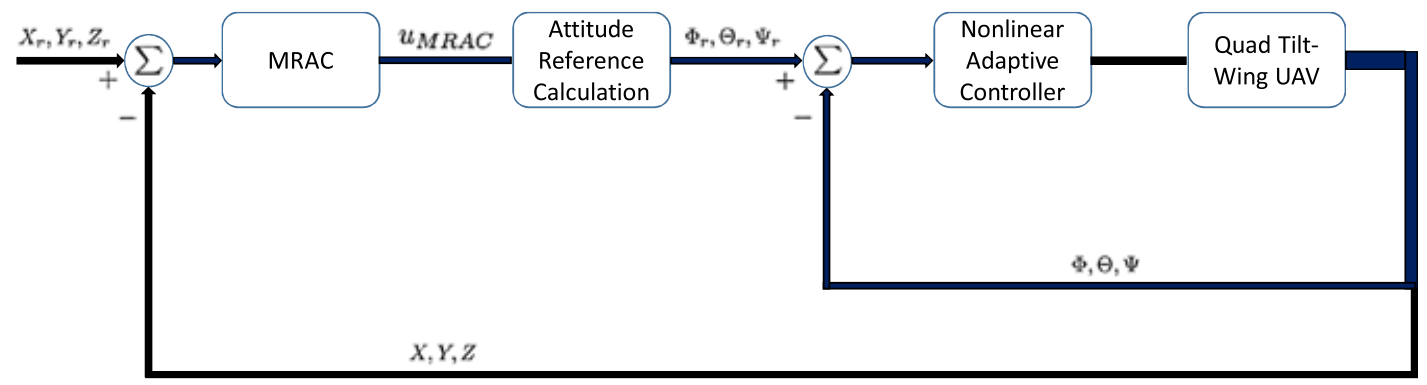

FIGURE 3 Closed-loop control system block diagram. MRAC, model reference adaptive control; UAV, unmanned aerial vehicle. [Colour figure can be 
the overall cascaded closed-loop system are investigated later in the paper.

Remark 2. The lift and drag coefficients required to compute the wing forces in Equation 20, $W_{l d}$, are obtained via nonlinear regression using the real data generated from wind-tunnel tests (see previous study ${ }^{38}$ ). In the controller design, these forces will be cancelled directly, and in the rest of the development, it will be assumed that this cancellation is already performed. The uncertainty occurring due to the computation of these forces are incorporated into the time-varying disturbance term $\pi(t)$.

\subsection{1 | Reference model design}

Consider the following control law, which is to be used for the nominal system dynamics, where $\Lambda=1, D=D_{n}=$ $\left[0_{1 \times 2} m_{n} g\right]^{T}$ and $\pi(t)=0$ :

$$
u_{n}=K_{x}^{T} \underline{X}+K_{r}^{T} r-D_{n},
$$

where $r \in \Re^{3}, K_{x} \in \mathfrak{R}^{6 \times 3}$, and $K_{r} \in R^{3 \times 3}$ are the reference input $\left(X_{r}, Y_{r}\right.$, and $\left.Z_{r}\right)$, control gain for the states, and control gain for the reference input, respectively. When Equation 26 is used for the nominal system, the nominal closed-loop dynamics is obtained, which is given below:

$$
\underline{X}_{n}=\left(A+B_{n} K_{x}^{T}\right) \underline{X}_{n}+B_{n} K_{r}^{T} r .
$$

In Equation 27, $K_{x}$ can be determined by any linear control design method, such as pole placement or linear quadratic regulator. Defining $A_{m}=A+B_{n} K_{x}^{T}$, nominal plant output is obtained as

$$
y_{n}=C\left(s I-A_{m}\right)^{-1} B_{n} K_{r}^{T} r .
$$

For a constant $r$, the steady-state plant output can be calculated as

$$
y_{s s}=-C A_{m}^{-1} B_{n} K_{r}^{T} r .
$$

Using $K_{r}^{T}=-\left(C A_{m}^{-1} B_{n}\right)^{-1}$, it is obtained that

$$
\lim _{t \rightarrow \infty}\left(y_{n}-r\right)=0 .
$$

As a result, the reference model dynamics is determined as

$$
\underline{\dot{X}}_{m}=A_{m} \underline{X}_{m}+B_{m} r,
$$

where

$$
A_{m}=A+B_{n} K_{x}^{T},
$$

and

$$
\begin{aligned}
B_{m} & =B_{n} K_{r}^{T} \\
& =-B_{n}\left(C A_{m}^{-1} B_{n}\right)^{-1} .
\end{aligned}
$$

\subsection{2 | Adaptive controller design}

When uncertainties are considered in the system dynamics (Equation 20), the fixed controller gains introduced in
Equation 26 must be replaced with their corresponding adaptive estimates:

$$
u_{M R A C}=\hat{K}_{x}^{T} \underline{X}+\hat{K}_{r}^{T} r+\hat{D},
$$

with the adaptive laws given below that can be shown to result in a stable closed-loop system, ${ }^{42,43}$

$$
\begin{aligned}
& \dot{\hat{K}}_{x}=-\Gamma_{x}\left(\underline{X} e^{T} P B_{n}+\sigma_{x}\|e\| \hat{K}_{x}\right), \\
& \dot{\hat{K}}_{r}=-\Gamma_{r}\left(r e^{T} P B_{n}+\sigma_{r}\|e\| \hat{K}_{r}\right), \\
& \dot{\hat{D}}^{T}=-\Gamma_{D}\left(e^{T} P B_{n}+\sigma_{D}\|e\| \hat{D}\right),
\end{aligned}
$$

where $e=X-X_{m}, \Gamma_{x} \in \Re^{6 \times 6}, \Gamma_{r} \in \mathfrak{R}^{3 \times 3}$, and $\Gamma_{D} \in \mathfrak{R}$ are adaptive gains, $\sigma_{x}, \sigma_{r}$, and $\sigma_{D}$ are positive scalar gains of e-modification terms, and $P \in \mathfrak{R}^{6 \times 6}$ is the symmetric solution of the Lyapunov equation

$$
A_{m}^{T} P+P A_{m}=-Q,
$$

where $Q \in \mathfrak{R}^{6 \times 6}$ is a positive definite matrix. However, it is noted that in this formulation, as mentioned earlier, it is assumed that the inner loop controller is perfect, and thus, no additional error terms appear in system dynamics due the transients of the inner loop. This assumption is violated in practice, and the attitude error dynamics from the inner loop will enter as additional disturbances to the dynamics provided in Equation 20. In the overall stability analysis of the cascaded control framework, it will be shown that with an additional quadratic e-modification robustifying term in one of the adaptive laws, the boundedness of all closed-loop system signals can be shown. The resulting adaptive laws are given as

$$
\begin{gathered}
\dot{\hat{K}}_{x}=-\Gamma_{x}\left(\underline{X} e^{T} P B_{n}+\sigma_{x}\|e\| \hat{K}_{x}+\gamma_{x}\|e\|^{2} \hat{K}_{x}\right), \\
\dot{\hat{K}}_{r}=-\Gamma_{r}\left(r e^{T} P B_{n}+\sigma_{r}\|e\| \hat{K}_{r}\right), \\
\dot{\hat{D}}^{T}=-\Gamma_{D}\left(e^{T} P B_{n}+\sigma_{D}\|e\| \hat{D}\right),
\end{gathered}
$$

where the term $\gamma_{x}$, used in the modified adaptive law (Equation 40), is a positive scalar. It is noted that the use of the newly added term " $\gamma_{x}\|e\|^{2} \hat{K}_{x}$ " will be clear in the overall stability analysis.

\section{2 | Attitude reference calculation}

Using the translational part (first 3 rows) of Equations 2 to 9 and incorporating the disturbance term $\pi(t)$, we obtain that

$$
\begin{gathered}
m \ddot{X}=\left(c_{\Psi} c_{\Theta} c_{\theta_{f}}-\left(c_{\Phi} s_{\Theta} c_{\Psi}+s_{\Phi} S_{\Psi}\right) s_{\theta_{f}}\right) u_{1}+W_{x}+\pi_{x}(t), \\
m \ddot{Y}=\left(s_{\Psi} c_{\Theta} c_{\theta_{f}}-\left(c_{\Phi} s_{\Theta} S_{\Psi}-s_{\Phi} c_{\Psi}\right) s_{\theta_{f}}\right) u_{1}+W_{y}+\pi_{y}(t), \\
m \ddot{Z}=\left(-s_{\Theta} c_{\theta_{f}}-c_{\Phi} c_{\Theta} s_{\theta_{f}}\right) u_{1}+m g+W_{z}+\pi_{z}(t),
\end{gathered}
$$

where $W_{x}, W_{y}$, and $W_{z}$ are the components of the lift-drag forces along 3 axes, which are given in Equation 14 . 
Similary, $\pi_{x}, \pi_{y}$, and $\pi_{z}$ are the components of the disturbance term along these axes. It is important to note that, as stated in Remark 2, the wing forces are computable and therefore will be canceled in the controller implementation. Accordingly, the applied control inputs will be calculated as $u_{M R A C}^{i}-\hat{W}_{j}$, where $i=1,2,3, j=x, y, z$, and $\hat{W}$ refers to the computed values of the wing forces. As seen from Equations 20 and 43 to 45 , these calculated control inputs will correspond to the projections of the total thrust $u_{1}$ on the $\mathrm{x}$-axis, $\mathrm{y}$-axis, and z-axis:

$$
\begin{gathered}
u_{M R A C}^{1}-\hat{W}_{x}=\left(c_{\Psi} c_{\Theta} c_{\theta_{f}}-\left(c_{\Phi} S_{\Theta} c_{\Psi}+s_{\Phi} S_{\Psi}\right) s_{\theta_{f}}\right) u_{1}, \\
u_{M R A C}^{2}-\hat{W}_{y}=\left(s \Psi c_{\Theta} c_{\theta_{f}}-\left(c_{\Phi} s_{\Theta} S_{\Psi}-s_{\Phi} c_{\Psi}\right) s_{\theta_{f}}\right) u_{1}, \\
u_{M R A C}^{3}-\hat{W}_{z}=\left(-s_{\Theta} c_{\theta_{f}}-c_{\Phi} c_{\Theta} s_{\theta_{f}}\right) u_{1} .
\end{gathered}
$$

It is important to note that the $D$ term in Equation 20 addresses the gravitational force $m g$. In light of Equations 14 and 15, it is clear that $W_{x}, W_{y}$, and $W_{z}$ (and therefore their computed values) are dependent on the attitude of the vehicle, ie,

$$
\left[\begin{array}{c}
W_{x} \\
W_{y} \\
W_{z}
\end{array}\right]=R_{b w}\left[\begin{array}{c}
F_{D}^{1}+F_{D}^{2}+F_{D}^{3}+F_{D}^{4} \\
0 \\
F_{L}^{1}+F_{L}^{2}+F_{L}^{3}+F_{L}^{4}
\end{array}\right],
$$

where the lift and drag forces, $F_{L}^{i}$ and $F_{D}^{i}$, are given in Equation 15.

The total thrust $u_{1}$ and the desired attitude angles $\Phi_{d}$ and $\Theta_{d}$ can be found using Equations 46 to 48 as

$$
\begin{gathered}
u_{1}=\sqrt{\left(u_{M R A C}^{1}-\hat{W}_{x}\right)^{2}+\left(u_{M R A C}^{2}-\hat{W}_{y}\right)^{2}+\left(u_{M R A C}^{3}-\hat{W}_{z}\right)^{2}}, \\
\Phi_{d}=\arcsin \left(\frac{-\rho_{1}}{u_{1} s_{\theta_{f}}}\right), \\
\Theta_{d}=\arcsin \left(\frac{-\left(u_{M R A C}^{3}-\hat{W}_{z}\right) u_{1} c_{\theta_{f}}-u_{1} \rho_{2} s_{\theta_{f}} c_{\Phi_{d}}}{\left(\rho_{2}\right)^{2}+\left(u_{M R A C}^{3}-\hat{W}_{z}\right)^{2}}\right),
\end{gathered}
$$

where

$$
\begin{aligned}
& \rho_{1}=\left(u_{M R A C}^{1}-\hat{W}_{x}\right) S_{\Psi_{d}}-\left(u_{M R A C}^{2}-\hat{W}_{y}\right) c_{\Psi_{d}}, \\
& \rho_{2}=\left(u_{M R A C}^{1}-\hat{W}_{x}\right) c_{\Psi_{d}}+\left(u_{M R A C}^{2}-\hat{W}_{y}\right) s_{\Psi_{d}} .
\end{aligned}
$$

One may obtain singular or degenerate configurations for certain $\Phi$ and $\Theta$ values, since both sides of Equations 46 to 48 are functions of the vehicle attitude due to the existence of lift and drag force terms. Since the flight trajectory of the vehicle in this study necessitates a VTOL motion followed by a transition to horizontal flight and then back to VTOL mode for landing, throughout the study and simulations, the solutions given by Equations 50 to 52 is used to calculate the total thrust and desired attitude angles. In addition, in simulation studies, we used hard limits for the desired attitude angles to prevent feeding excessively large angles as references to the inner control loop. We leave the singular or degenerate configurations for which $\Phi_{d}$ and $\Theta_{d}$ can not be obtained uniquely as a future work.

It is noted that, different from similar works in the literature, the desired attitude angles are functions of the wing angles. The desired yaw angle, $\Psi_{d}$, can be chosen by the UAV operator that would be appropriate for the mission at hand. These required attitude angles are given to the lower-level attitude controller as references. The nonlinear adaptive attitude controller is described in the next section.

\section{3 | Nonlinear adaptive control design}

To force the UAV to follow the requested attitude angles, in the presence of uncertainties, we used a nonlinear adaptive controller similar to 1 study. ${ }^{44}$ Defining $u^{\prime}=E^{T} M_{t}$, Equation 17 can be rewritten as

$$
M\left(\alpha_{w}\right) \dot{\Omega}_{w}+C\left(\alpha_{w}, \Omega_{w}\right) \Omega_{w}=u^{\prime} .
$$

Equation 55, which describes the rotational dynamics of the vehicle, can be parameterized in a way such that the vector consisting of the diagonal elements of the moment of inertia of the UAV, $I_{U A V}=\left[I_{x x}, I_{y y}, I_{z z}\right]^{T}$, appears linearly. This transformation is needed so that the uncertain moment of inertia terms appears in a form that is suitable for the adaptive control design:

$$
Y\left(\alpha_{w}, \dot{\alpha}_{w}, \ddot{\alpha}_{w}\right) I_{U A V}=u^{\prime} .
$$

Consider the following definition

$$
s=\dot{\tilde{\alpha}}_{w}+\Lambda_{s} \tilde{\alpha}_{w},
$$

where $\tilde{\alpha}_{w}=\alpha_{w}-\alpha_{w d}, \alpha_{w d}$ is the desired value of $\alpha_{w}$ and $\Lambda_{s} \in$ $\mathfrak{R}^{3 \times 3}$ is a symmetric positive definite matrix. Equation 57 can be modified as

$$
s=\dot{\alpha}_{w}-\dot{\alpha}_{w r},
$$

where

$$
\dot{\alpha}_{w r}=\dot{\alpha}_{w d}-\Lambda_{s} \tilde{\alpha}_{w} .
$$

A matrix $Y^{\prime}=Y^{\prime}\left(\alpha_{w}, \dot{\alpha}_{w}, \dot{\alpha}_{w r}, \ddot{\alpha}_{w r}\right)$ can be defined, to be used in linear parameterization of Equation 55, as in the case of Equation 56, such that

$$
M\left(\alpha_{w}\right) \ddot{\alpha}_{w r}+C\left(\alpha_{w}, \Omega_{w}\right) \dot{\alpha}_{r}=Y^{\prime}\left(\alpha_{w}, \dot{\alpha}_{w}, \dot{\alpha}_{w r}, \ddot{\alpha}_{w r}\right) I_{U A V} .
$$

It can be shown that the following nonlinear controller,

$$
u_{N a d p} \equiv u^{\prime}=Y^{\prime} \hat{\mathrm{I}}_{U A V}-K_{D} s,
$$

where $K_{D} \in \mathfrak{R}^{3 \times 3}$ is positive definite matrix and $\hat{\mathrm{I}}_{U A V}$ is an estimate of the uncertain parameter $I$, with an adaptive law

$$
\dot{\hat{\mathrm{I}}}_{U A V}=-\Gamma_{I} Y^{\prime T} s,
$$

where $\Gamma_{I}$ is the adaptation rate, stabilizes the rotational closed-loop system, and makes the error $\tilde{\alpha}_{w}$ converge to 0 . 
The total thrust $u_{1}$ is provided in Equation 50. The rest of the control inputs in Equation 9 can be calculated as in the previous study ${ }^{37}$ by first defining $u^{\prime \prime}=\left(E\left(\alpha_{w}\right)^{T}\right)^{-1} u^{\prime}$ and performing the following operations:

$$
\begin{gathered}
u_{3}=\frac{u_{2}^{\prime \prime}}{s_{\theta_{f}}}, \\
{\left[\begin{array}{l}
u_{2} \\
u_{4}
\end{array}\right]=\left[\begin{array}{cc}
s_{\theta_{f}} & -c_{\theta_{f}} \\
c_{\theta_{f}} & s_{\theta_{f}}
\end{array}\right]^{-1}\left[\begin{array}{l}
u_{1}^{\prime \prime} \\
u_{3}^{\prime \prime}
\end{array}\right] .}
\end{gathered}
$$

Once these control inputs are determined, the thrusts created by the rotors can be calculated using linear relationships given in Equations 10 to 13.

Remark 3. The required translational and rotational states of the UAV can be obtained by the help of several onboard sensors, such as Global Positioning System (GPS) antennas and receivers and Inertial Measurement Unit (IMU) units, processors, and algorithms, which form a full GPS Inertial Navigation System. It is noted that no state observer that needs exact plant dynamics is assumed to be available for the development of the proposed control algorithm. The information about the details of the UAV state measurements and estimation can be obtained from the literature. ${ }^{45}$

\section{4 | Overall closed-loop stability}

In this section, the stability of the tilt-wing UAV together with the nonlinear hierarchical adaptive controller is investigated. Before starting this investigation, system dynamics is converted to a more suitable form for stability analysis, below.

Consider the closed-loop dynamics given in Equation 20, where it is assumed that the UAV is a simple mass and is controlled via the control input $u_{M R A C}$ in the presence of parametric uncertainty, lift and drag forces, gravity, and unknown external disturbances. (Note that in the implementation, the computed lift and drag forces are canceled in the control signal and the uncertainty in this computation is incorporated in the disturbance term, as stated in Remark 2.) Adaptive control input $u_{M R A C}$ is designed based on this assumption. This assumption is valid except that the realization of $u_{M R A C}$ is imperfect: The inner loop controller is used to achieve the necessary attitude angles that would result in the realization of $u_{M R A C}$, and since the inner loop controller does not have infinite bandwidth, $u_{M R A C}$ is realized with certain errors. Specifically, introducing attitude-tracking errors in Equation 46 to 48 , the realization of $u_{M R A C}$ is given as

$$
\begin{aligned}
& u_{M R A C}^{1}=\left(c_{\Psi_{d}+e_{\Psi}} c_{\Theta_{d}+e_{\Theta}} c_{\Theta_{f}}-\right. \\
& \left.\left(c_{\Phi_{d}+e_{\Phi}} s_{\Theta_{d}+e_{\Theta}} c \Psi_{d}+e_{\Psi}+s_{\Phi_{d}+e_{\Phi}} s \Psi_{d}+e_{\Psi}\right) s_{\theta_{f}}\right) u_{1}, \\
& u_{M R A C}^{2}=\left(s \Psi_{d}+e_{\Psi} c_{\Theta_{d}+e_{\Theta}} c_{\theta_{f}}\right. \\
& \left.-\left(c_{\Phi_{d}+e_{\Phi}} s_{\Theta_{d}+e_{\Theta}} S_{\Psi_{d}+e_{\Psi}}-s_{\Phi_{d}+e_{\Phi}} c_{\Psi_{d}+e_{\Psi}}\right) s_{\theta_{f}}\right) u_{1},
\end{aligned}
$$

$$
u_{M R A C}^{3}=\left(-s_{\Theta_{d}+e_{\Theta}} c_{\theta_{f}}-c_{\Phi_{d}+e_{\Phi}} c_{\Theta+e_{\Theta}} s_{\theta_{f}}\right) u_{1},
$$

where $\Phi_{d}, \Theta_{d}$, and $\Psi_{d}$ are the desired attitude angles and $e_{\Phi}$, $e_{\Theta}$, and $e_{\Psi}$ are the attitude errors. It is noted that there is no error term introduced to $u_{1}$ since the calculated desired thrust is directly used without further realization by the inner loop controller.

Consider the following trigonometric relationships:

$$
\begin{aligned}
& \sin (a+b)=\sin (a)+\sin (b / 2) \cos (a+b / 2), \\
& \cos (a+b)=\cos (a)-\sin (b / 2) \sin (a+b / 2) .
\end{aligned}
$$

Using Equation 68 and following the same procedure given in another study, ${ }^{46}$ Equations 65 to 67 can be rewritten as

$$
\begin{aligned}
u_{M R A C}^{1}= & \left(c_{\Psi_{d}} c_{\Theta_{d}} c_{\theta_{f}}-\left(c_{\Phi_{d}} s_{\Theta_{d}} c_{\Psi_{d}}+s_{\Phi_{d}} s \Psi_{d}\right) s_{\theta_{f}}\right. \\
& \left.+g_{1}\left(\Phi_{d}, \Theta_{d}, \Psi_{d}, e_{\Phi}, e_{\Theta}, e_{\Psi}, \theta_{f}\right)\right) u_{1} \\
= & u_{M d}^{1}+u_{1} g_{1}(.) \\
u_{M R A C}^{2}= & \left(s_{\Psi_{d}} c_{\Theta_{d}} c_{\theta_{f}}-\left(c_{\Phi_{d}} s_{\Theta_{d}} s \Psi_{d}-s_{\Phi_{d}} c_{\Psi_{d}}\right) s_{\theta_{f}}\right. \\
& \left.+g_{2}\left(\Phi_{d}, \Theta_{d}, \Psi_{d}, e_{\Phi}, e_{\Theta}, e_{\Psi}, \theta_{f}\right)\right) u_{1} \\
= & u_{M d}^{2}+u_{1} g_{2}(.) \\
u_{M R A C}^{3}= & \left(-S_{\Theta_{d}} c_{\theta_{f}}-c_{\Phi_{d}} c_{\Theta_{d}} s_{\theta_{f}}+g_{3}\left(\Phi_{d}, \Theta_{d}, e_{\Phi}, e_{\Theta}, \theta_{f}\right)\right) u_{1} \\
= & u_{M d}^{3}+u_{1} g_{3}(.)
\end{aligned}
$$

where the terms $u_{M d}^{i}, i=1,2$, and 3 , refer to the ith desired MRAC control input and the terms $u_{1} g_{i}($.$) are the errors,$ which consist of sine and cosine functions of the desired attitude angles, attitude errors, and the front wing angle, in the realization of the control inputs. Defining $u_{M R A C}^{d}=$ $\left[\begin{array}{lll}u_{M d}^{1} & u_{M d}^{2} & u_{M d}^{3}\end{array}\right]^{T}$ and using Equations 69 to 71 , and noting that the total thrust $u_{1}$ is equal to $\left\|u_{M R A C}^{d}\right\|$, Equation 20 can be rewritten as

$\underline{\dot{X}}=A \underline{X}+B_{n} \Lambda\left(u_{M R A C}^{d}+\left\|u_{M R A C}^{d}\right\|\left[\begin{array}{l}g_{1}(.) \\ g_{2}(.) \\ g_{3}(.)\end{array}\right]+D+\pi(t)\right)$.

The following lemma, regarding an upper bound on $\left\|u_{M R A C}^{d}\right\|$, will be useful in providing a proof for the main theorem of this paper.

Lemma 1. For a bounded reference $r,\left\|u_{M R A C}^{d}\right\| \leqslant c_{1}+$ $c_{2}\|e\|+c_{3}\left\|\tilde{K}_{x}\right\|+\left\|\tilde{K}_{x}\right\|\|e\|+c_{4}\left\|\tilde{K}_{r}\right\|+\|\tilde{D}\|$, where $c_{i}$ are positive scalars, $e \in \mathfrak{R}^{6}$ is the error vector between the reference model states and the system states, and

$$
\begin{aligned}
\tilde{K}_{x} & =\hat{K}_{x}-K_{x}^{*}, \\
\tilde{K}_{r} & =\hat{K}_{r}-K_{r}^{*}, \\
\tilde{D} & =\hat{D}-D^{*},
\end{aligned}
$$

with $(.)^{*}$ being the "ideal value" and (.) being the estimated value of a parameter.

It is noted that here and in the rest of the paper, Frobenius norm is used. 
Remark 4. As explained earlier, the control signal given in Equation 35 is the desired control input provided by the position controller. Since in the controller development, it was assumed that this signal is realized perfectly; the superscript "d" was not used. In the stability proof, however, the position control input is represented with $u_{M R A C}^{d}$, which removes this assumption.

Proof. (Proof of lemma 1)

Using Equation 73, Equation 35 can be rewritten as

$$
u_{M R A C}^{d}=K_{x}^{*^{T}} X+\tilde{K}_{x} X+K_{r}^{*^{T}} r+\tilde{K}_{r} r+D^{*}+\tilde{D} .
$$

Using the fact that $e=X-X_{m}$, Equation 74 can be rewritten as $u_{M R A C}^{d}=K_{x}^{*^{T}} X_{m}+K_{x}^{*^{T}} e+\tilde{K}_{x} X_{m}+\tilde{K}_{x} e+K_{r}^{*^{T}} r+\tilde{K}_{r} r+D^{*}+\tilde{D}$.

Taking the norm of both sides of Equation 75 and using the triangular inequality, it is obtained that

$$
\begin{aligned}
\left\|u_{M R A C}^{d}\right\| \leqslant & \left\|K _ { x } ^ { * ^ { T } } \left|\left\||| X_{m}\right\|+\left\|K_{x}^{*^{T}}\left|\|\| e\|+\| \tilde{K}_{x}\|\|\right| X_{m}\right\|\right.\right. \\
& +\left\|\tilde{K}_{x}\right\|\left\|| | \left|+\left\|K_{r}^{*^{T}}\right\|\|r\|+\left\|\tilde{K}_{r}\right\|\||| r\|\right.\right. \\
& +\left\|D^{*}\right\|+\|\tilde{D}\| .
\end{aligned}
$$

$K_{x}^{*}, K_{r}^{*}$, and $D^{*}$ are constant matrices. Also, it is known that the states of the reference model $X_{m}$ are bounded. Defining $\left\|K_{x}^{*}\right\| \equiv k_{1},\left\|K_{r}^{*}\right\| \equiv k_{2},\left\|D^{*}\right\| \equiv k_{3},\|r\| \equiv k_{4}$, and $\left\|X_{m}\right\| \equiv$ $k_{5}$, Equation 76 can be rewritten as

$$
\begin{aligned}
\left\|u_{M R A C}^{d}\right\| & \leqslant k_{1} k_{5}+k_{1}\|e\|+k_{5}\left\|\tilde{K}_{x}\right\|+\left\|\tilde{K}_{x}\right\| \||| \mid+k_{2} k_{4} \\
& +k_{4}\left\|\tilde{K}_{r}\right\|+k_{3}+\|\tilde{D}\| .
\end{aligned}
$$

Defining $c_{1} \equiv k_{1} k_{5}+k_{2} k_{4}+k_{3}, c_{2} \equiv k_{1}, c_{3} \equiv k_{5}$, and $c_{4} \equiv k_{4}$, it is obtained that

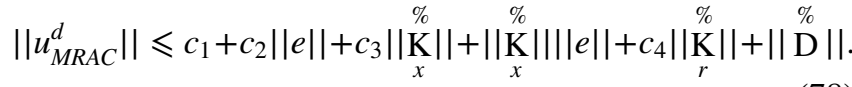

This completes the proof of Lemma 1.

The following lemma will also be instrumental in proving the main theorem of this work.

Lemma 2. $\bar{G} \leqslant k\left\|\tilde{\alpha}_{\omega}\right\|$, where $\tilde{\alpha}_{\omega}$ is the attitude-tracking error vector and $k$ is a positive constant.

Remark 5. A classical hierarchical controller is designed in previous study ${ }^{36}$ for a similar plant, where the errors introduced by the inner loop controller is investigated. It is noted that regardless of the controller type, the same error terms will appear as disturbances in the outer loop controller. A proof of Lemma 2 is given in another study, ${ }^{36}$ and thus, the proof is omitted here.

Theorem 1. All the signals in the closed-loop system, consisting of the UAV dynamics (Equation 72), the reference model (Equation 31), MRAC (Equations 35-42), and nonlinear adaptive controller (Equations 61 and 62), are uniformly ultimately bounded (UUB), which indicates that the bound on the signals does not depend on the initial time $t_{0}$ and that this uniform bound holds after a certain time T, ie, for $t \geqslant t_{0}+T$. It is noted that in UUB, unlike Lyapunov stability, the bound on the signals cannot be made arbitrarily small with proper initial conditions. In practice, this bound is a function of uncertainties and disturbances. ${ }^{42}$ In addition, defining $G()=.\left[g_{1}(.) g_{2}(.) g_{3}(.)\right]^{T}$, the following are true:

$$
\lim _{t \rightarrow \infty} G(.)=\underline{0},
$$

$\sup \|G().\| \leqslant k|| \Lambda_{s}^{-1} \|$

$$
\times\left(\left(K-\lambda_{\min }\left(\Gamma^{-1}\right) \times\left\|\tilde{I}_{U A V}\right\|^{2}\right) / \bar{\lambda}_{\max }(M)\right)^{1 / 2},
$$

where $0 \in \mathfrak{R}^{3}$ is a vector of zeros, $k \in \mathfrak{R}^{+}$is a constant, "sup" refers to "supremum," $\bar{\lambda}_{\max }$ is the bound on the maximum eigenvalue of $M$, and

$$
K=\frac{1}{2}\left(s(0)^{T} M(0) s(0)+\tilde{I}_{U A V}(0)^{T} \Gamma_{I}^{-1} \tilde{I}_{U A V}(0)\right) .
$$

Proof. (Proof of Theorem 1:)

In light of Remark 4, substituting the control input Equation 35 into Equation 72, it is obtained that

$$
\begin{aligned}
\underline{\dot{X}}= & \left(A+B_{n} \Lambda \hat{K}_{x}^{T}\right) \underline{X} \\
& +B_{n} \Lambda\left(\hat{K}_{r}^{T} r+\hat{D}+\left\|u_{M R A C}^{d}\right\|\left[\begin{array}{l}
g_{1}(.) \\
g_{2}(.) \\
g_{3}(.)
\end{array}\right]+D+\pi(t)\right) .
\end{aligned}
$$

Assuming that there exist $K_{x}^{*}$ and $K_{r}^{*}$ such that

$$
\begin{aligned}
A+B_{n} \Lambda K_{x}^{*^{T}} & =A_{m}, \\
B_{n} \Lambda K_{r}^{*^{T}} & =B_{m},
\end{aligned}
$$

and defining $\hat{K}_{x}=K_{x}^{*}+\tilde{K}_{x}, \hat{K}_{r}=K_{r}^{*}+\tilde{K}_{r}$ and $\hat{D}=D^{*}+\tilde{D}=$ $-D+\tilde{D}$, Equation 82 can be rewritten as

$$
\begin{aligned}
\underline{\dot{X}}= & A_{m} \underline{X}+B_{m} r+B_{n} \Lambda \tilde{K}_{x}^{T} \underline{X}+B_{n} \Lambda \tilde{K}_{r}^{T} r \\
& +B_{n} \Lambda \tilde{D}+B_{n} \Lambda\left\|u_{M R A C}^{d}\right\|\left[\begin{array}{l}
g_{1}(.) \\
g_{2}(.) \\
g_{3}(.)
\end{array}\right]+B_{n} \Lambda \pi(t) .
\end{aligned}
$$

Subtracting the reference model Equation 31 from Equation 84, it is obtained that

$$
\begin{aligned}
\dot{e}= & A_{m} e+B_{n} \Lambda \tilde{K}_{x}^{T} \underline{X}+B_{n} \Lambda \tilde{K}_{r}^{T} r+B_{n} \Lambda \tilde{D} \\
& +B_{n} \Lambda\left\|u_{M R A C}^{d}\right\|\left[\begin{array}{l}
g_{1}(.) \\
g_{2}(.) \\
g_{3}(.)
\end{array}\right]+B_{n} \Lambda \pi(t),
\end{aligned}
$$

where $e=X-X_{m}$.

Consider the following Lyapunov function candidate

$$
V=e^{T} P e+\operatorname{tr}\left(\left[\tilde{K}_{x}^{T} \Gamma_{x}^{-1} \tilde{K}_{x}+\tilde{K}_{r}^{T} \Gamma_{r}^{-1} \tilde{K}_{r}+\tilde{D}^{T} \Gamma_{D}^{-1} \tilde{D}\right] \Lambda\right),
$$

where "tr" refers to the trace operation and $P$ is a symmetric positive definite matrix that is the solution of the Lyapunov equation 


$$
A_{m}^{T} P+P A_{m}=-Q
$$

where $Q \in \mathfrak{R}^{6 \times 6}$ is a symmetric positive definite matrix. Taking the derivative of both sides of Equation 86, it is obtained that

$$
\begin{aligned}
\dot{V}= & \dot{e}^{T} P e+e^{T} P \dot{e} \\
& +2 \operatorname{tr}\left(\left[\tilde{K}_{x}^{T} \Gamma_{x}^{-1} \dot{\tilde{K}}_{x}+\tilde{K}_{r}^{T} \Gamma_{r}^{-1} \dot{\tilde{K}}_{r}+\tilde{D}^{T} \Gamma_{D}^{-1} \dot{\tilde{D}}\right] \Lambda\right) \\
= & \left(e^{T} A_{m}^{T}+\underline{X}^{T} \tilde{K}_{x} \Lambda B_{n}^{T}+r^{T} \tilde{K}_{r} \Lambda B_{n}^{T}+\tilde{D}^{T} \Lambda B_{n}^{T}\right. \\
& \left.+G(.)^{T}\left\|u_{M R A C}^{d}\right\| \Lambda B_{n}^{T}+\pi(t)^{T} \Lambda B_{n}^{T}\right) P e \\
& +e^{T} P\left(A_{m} e+B_{n} \Lambda \tilde{K}_{x}^{T} \underline{X}+B_{n} \Lambda \tilde{K}_{r}^{T} r+B_{n} \Lambda \tilde{D}\right. \\
& \left.+B_{n} \Lambda\left\|u_{M R A C}^{d}\right\| G(.)+B_{n} \Lambda \pi(t)\right) \\
& +2 \operatorname{tr}\left(\left[\tilde{K}_{x}^{T} \Gamma_{x}^{-1} \dot{\tilde{K}}_{x}+\tilde{K}_{r}^{T} \Gamma_{r}^{-1} \dot{\tilde{K}}_{r}+\tilde{D}^{T} \Gamma_{D}^{-1} \dot{\tilde{D}}\right] \Lambda\right) \\
= & e^{T}\left(A_{m}^{T} P+P A_{m}\right) e+2 e^{T} P B_{n} \Lambda\left(\tilde{K}_{x}^{T} \underline{X}+\tilde{K}_{r}^{T} r+\tilde{D}\right. \\
& \left.+\left\|u_{M R A C}^{d}\right\| G(.)+\pi(t)\right) \\
& +2 \operatorname{tr}\left(\left[\tilde{K}_{x}^{T} \Gamma_{x}^{-1} \tilde{\tilde{K}}_{x}+\tilde{K}_{r}^{T} \Gamma_{r}^{-1} \dot{\tilde{K}}_{r}+\tilde{D}^{T} \Gamma_{D}^{-1} \dot{\tilde{D}}\right] \Lambda\right) \\
= & -e^{T} Q e+2 e^{T} P B_{n} \Lambda \tilde{K}_{x}^{T} \underline{X}+2 \operatorname{tr}\left(\left[\tilde{K}_{x}^{T} \Gamma_{x}^{-1} \dot{\tilde{K}}_{x}\right] \Lambda\right) \\
& +2 e^{T} P B_{n} \Lambda \tilde{K}_{r}^{T} r+2 \operatorname{tr}\left(\left[\tilde{K}_{r}^{T} \Gamma_{r}^{-1} \dot{\tilde{K}}_{r}\right] \Lambda\right)+2 e^{T} P B_{n} \Lambda \tilde{D} \\
& +2 \operatorname{tr}\left(\left[\tilde{D}^{T} \Gamma_{D}^{-1} \dot{\tilde{D}}\right] \Lambda\right)+2 e^{T} P B_{n} \Lambda\left\|u_{M R A C}^{d}\right\| G(.) \\
& +2 e^{T} P B_{n} \Lambda \pi(t),
\end{aligned}
$$

where $G()=.\left[g_{1}(.) g_{2}(.) g_{3}(.)\right]^{T}$. Using the property of the trace operator, $a^{T} b=\operatorname{tr}\left(b a^{T}\right)$, where $a$ and $b$ are vectors, Equation 88 can be rewritten as

$$
\begin{aligned}
\dot{V}= & -e^{T} Q e+2 \operatorname{tr}\left(\left[\tilde{K}_{x}^{T} \Gamma_{x}^{-1} \dot{\tilde{K}}_{x}+\tilde{K}_{x}^{T} \underline{X} e^{T} P B_{n}\right] \Lambda\right) \\
& +2 \operatorname{tr}\left(\left[\tilde{K}_{r}^{T} \Gamma_{r}^{-1} \dot{\tilde{K}}_{r}+\tilde{K}_{r}^{T} r e^{T} P B_{n}\right] \Lambda\right) \\
& +2 \operatorname{tr}\left(\left[\tilde{D}^{T} \Gamma_{D}^{-1} \dot{\tilde{D}}+\tilde{D}^{T} e^{T} P B_{n}\right] \Lambda\right) \\
& +2 e^{T} P B_{n} \Lambda\left\|u_{M R A C}^{d}\right\| G(.)+2 e^{T} P B_{n} \Lambda \pi(t) .
\end{aligned}
$$

Substituting Equations 40 to 42 in Equation 89, it is obtained that

$$
\begin{aligned}
\dot{V}= & -e^{T} Q e+2 \operatorname{tr}\left(\left[-\sigma_{x} \tilde{K}_{x}^{T}\|e\| \hat{K}_{x}-\gamma_{x} \tilde{K}_{x}^{T}\|e\|^{2} \hat{K}_{x}\right] \Lambda\right) \\
& +2 \operatorname{tr}\left(-\sigma_{r} \tilde{K}_{r}^{T}\|e\| \hat{K}_{r} \Lambda\right)+2 \operatorname{tr}\left(-\sigma_{D} \tilde{D}^{T}\|e\| \hat{D} \Lambda\right) \\
& +2 e^{T} P B_{n} \Lambda\left\|u_{M R A C}^{d}\right\| G(.)+2 e^{T} P B_{n} \Lambda \pi(t) \\
\leqslant & -\|e\|^{2} \lambda_{\min }(Q)+2 \operatorname{tr}\left(\left[-\sigma_{x} \tilde{K}_{x}^{T}\|e\| \hat{K}_{x}-\gamma_{x} \tilde{K}_{x}^{T}\|e\|^{2} \hat{K}_{x}\right.\right. \\
& \left.\left.-\sigma_{r} \tilde{K}_{r}^{T}\|e\| \hat{K}_{r}-\sigma_{D} \tilde{D}^{T}\|e\| \hat{D}\right] \Lambda\right) \\
& +2\|e\|\left\|P B_{n} \Lambda\right\|\left\|u_{M R A C}^{d}\right\|\|G(.)\|+2\|e\|\left\|P B_{n} \Lambda\right\| \bar{\pi}
\end{aligned}
$$

where $\lambda_{\min }(Q)$ refers to the minimum eigenvalue of the matrix $Q$ and $\bar{\pi}$ is the upper bound of $\pi(t)$. Using the fact that the norm of the vector $G($.) is bounded by a constant $\bar{G}$, since the vector terms consist of sines and cosines, and using Lemma 1, it is obtained from Equation 90 that

$$
\begin{aligned}
\dot{V} \leqslant & -\|e\|^{2} \lambda_{\min }(Q)+2 \operatorname{tr}\left(\left[-\sigma_{x} \tilde{K}_{x}^{T}\|e\| \hat{K}_{x}-\gamma_{x} \tilde{K}_{x}^{T}\|e\|^{2} \hat{K}_{x}\right.\right. \\
& \left.\left.-\sigma_{r} \tilde{K}_{r}^{T}\|e\| \hat{K}_{r}-\sigma_{D} \tilde{D}^{T}\|e\| \mid \hat{D}\right] \Lambda\right) \\
& +2 \bar{G}\|e\|\left\|P B_{n} \Lambda\right\|\left(c_{1}+c_{2}\|e\|+c_{3}\left\|\tilde{K}_{x}\right\|+\left\|\tilde{K}_{x}\right\|\|e\|\right. \\
& \left.+c_{4}\left\|\tilde{K}_{r}\right\|+\|\tilde{D}\|\right)+2\|e\|\left\|P B_{n} \Lambda\right\| \bar{\pi} .
\end{aligned}
$$

Completing the square, the first term in the trace operation parenthesis can be rewritten as follows:

$$
\begin{aligned}
& \operatorname{tr}\left(\left[-\sigma_{x} \tilde{K}_{x}^{T}\|e\| \hat{K}_{x}\right] \Lambda\right) \leqslant \lambda_{\min }(\Lambda) \times \operatorname{tr}\left(-\sigma_{x} \tilde{K}_{x}^{T}\|e\|\left(K_{x}^{*}+\tilde{K}_{x}\right)\right) \\
& \quad=-\sigma_{x}\|e\| \lambda_{\min }(\Lambda)\left(\frac{K_{x}^{*}}{2}+\tilde{K}_{x}{ }^{2}-\frac{K_{x}^{* 2}}{2}\right) \\
& \quad \leqslant-\sigma_{x}\|e\| \lambda_{\min }(\Lambda)\left(\tilde{K}_{x}{ }^{2}-K_{x}^{* 2}\right) .
\end{aligned}
$$

Using the same procedure, Equation 91 can be rewritten as

$$
\begin{aligned}
\dot{V} \leqslant & -\|e\|^{2} \lambda_{\min }(Q)-\sigma_{x}\|e\| \lambda_{\min }(\Lambda) \tilde{K}_{x}{ }^{2} \\
& +\sigma_{x}\|e\| \lambda_{\min }(\Lambda) K_{x}^{* 2} \\
& -\gamma_{x}\|e\|^{2} \lambda_{\min }(\Lambda) \tilde{K}_{x}^{2}+\gamma_{x}\|e\|^{2} \lambda_{\min }(\Lambda) K_{x}^{* 2} \\
& -\sigma_{r}\|e\| \lambda_{\min }(\Lambda) \tilde{K}_{r}^{2}+\sigma_{r}\|e\| \lambda_{\min }(\Lambda) K_{r}^{* 2} \\
& -\sigma_{D}\|e\| \lambda_{\min }(\Lambda) \tilde{D}^{2}+\sigma_{D}\|e\| \lambda_{\min }(\Lambda) D^{* 2} \\
& +2 \bar{G}\|e\|\left\|P B_{n} \Lambda\right\|\left(c_{1}+c_{2}\|e\|+c_{3}\left\|\tilde{K}_{x}\right\|+\left\|\tilde{K}_{x}\right\|\|e\|\right. \\
& \left.+c_{4}\left\|\tilde{K}_{r}\right\|+\|\tilde{D}\|\right)+2\|e\|\left\|P B_{n} \Lambda\right\| \bar{\pi} \\
= & -\|e\|\left(\| e \| \left\{\lambda_{\min }(Q)+\gamma_{x} \lambda_{\min }(\Lambda) \tilde{K}_{x}{ }^{2}\right.\right. \\
& -\gamma_{x} \lambda_{\min }(\Lambda) K_{x}^{* 2}-2 \bar{G}\left\|P B_{n} \Lambda\right\|\left\|\tilde{K}_{x}\right\| \\
& \left.-2 \bar{G}\left\|P B_{n} \Lambda\right\| c_{2}\right\}+\sigma_{x} \lambda_{\min }(\Lambda) \tilde{K}_{x}{ }^{2}-\sigma_{x} \lambda_{\min }(\Lambda) K_{x}^{* 2} \\
& +\sigma_{r} \lambda_{\min }(\Lambda) \tilde{K}_{r}{ }^{2}-\sigma_{r} \lambda_{\min }(\Lambda) K_{r}^{* 2}+\sigma_{D} \lambda_{\min }(\Lambda) \tilde{D}^{2} \\
& -\sigma_{D} \lambda_{\min }(\Lambda) D^{* 2}-2 \bar{G}\left\|P B_{n} \Lambda\right\| c_{1} \\
& -2 \bar{G}\left\|P B_{n} \Lambda\right\| c_{3}\left\|\tilde{K}_{x}\right\|-2 \bar{G}\left\|P B_{n} \Lambda\right\| c_{4}\left\|\tilde{K}_{r}\right\| \\
& \left.-2 \bar{G}\left\|P B_{n} \Lambda\right\|\|\tilde{D}\|-2\left\|P B_{n} \Lambda\right\| \bar{\pi}\right) .
\end{aligned}
$$

Assume that there exists an $\varepsilon>0$ such that

$$
\begin{aligned}
& \lambda_{\min }(Q)+\gamma_{x} \lambda_{\min }(\Lambda) \tilde{K}_{x}{ }^{2}-\gamma_{x} \lambda_{\min }(\Lambda) K_{x}^{*}{ }^{2}-2 \bar{G}|| P B_{n} \Lambda\|\| \tilde{K}_{x} \| \\
& \quad-2 \bar{G}\left\|P B_{n} \Lambda\right\| c_{2}-\varepsilon>0 .
\end{aligned}
$$

Using Equation 94, it can be concluded that $\dot{V} \leqslant 0$ in the complement of the set $S$, where $S$ is defined as

$$
\begin{aligned}
S \equiv & \left\{\left(e, \tilde{K}_{x}, \tilde{K}_{r}\right) \mid \varepsilon\|e\|+\sigma_{x} \lambda_{\min }(\Lambda) \tilde{K}_{x}{ }^{2}\right. \\
& -\sigma_{x} \lambda_{\min }(\Lambda) K_{x}^{* 2}+\sigma_{r} \lambda_{\min }(\Lambda) \tilde{K}_{r}{ }^{2} \\
& -\sigma_{r} \lambda_{\min }(\Lambda) K_{r}^{* 2}+\sigma_{D} \lambda_{\min }(\Lambda) \tilde{D}^{2} \\
& -\sigma_{D} \lambda_{\min }(\Lambda) D^{* 2}-2 \bar{G}|| P B_{n} \Lambda \| c_{1} \\
& -2 \bar{G}|| P B_{n} \Lambda\left\|c_{3}\right\| \tilde{K}_{x}\|-2 \bar{G}\| P B_{n} \Lambda\left\|c_{4}\right\| \tilde{K}_{r} \| \\
& -2 \bar{G}\left\|P B_{n} \Lambda\left|\||| \tilde{D}\|-2\left\|P B_{n} \Lambda\right\| \bar{\pi} \leqslant 0\right\} .\right.
\end{aligned}
$$


Therefore, using standard arguments, it can be shown ${ }^{43}$ that all the solutions are UBB. This completes the proof of the first part of Theorem 1. It is noted that the inclusion of the quadratic e-modification robustifying term in Equation 40 is beneficial in obtaining a negative semidefinite Lyapunov function derivative.

It is noted that the assumed system property (Equation 94) can be investigated further by completing the square and rewriting the inequality as

$$
\begin{aligned}
\lambda_{\min }(Q) & +\left(\sqrt{\gamma_{x} \lambda_{\min }(\Lambda)} \tilde{K}_{x}-\frac{\bar{G}\left\|P B_{n} \Lambda\right\|}{\sqrt{\gamma_{x} \lambda_{\min }(\Lambda)}}\right)^{2} \\
& -\gamma_{x} \lambda_{\min }(\Lambda) K_{x}^{* 2}-\frac{\bar{G}^{2}\left\|P B_{n} \Lambda\right\|^{2}}{\gamma_{x} \lambda_{\min }(\Lambda)} \\
& -2 \bar{G}|| P B_{n} \Lambda \| c_{2}-\varepsilon>0 .
\end{aligned}
$$

A sufficient condition to satisfy this inequality is given as

$$
\lambda_{\text {min }}(Q)-\gamma_{x} \lambda_{\min }(\Lambda) K_{x}^{* 2}>\frac{\bar{G}^{2}\left\|P B_{n} \Lambda\right\|^{2}}{\gamma_{x} \lambda_{\min }(\Lambda)}+2 \bar{G}\left\|P B_{n} \Lambda\right\| c_{2}+\varepsilon .
$$

Remark 6. Both the bound on the tracking error in Equation 95 and the restriction on system parameters given in Equation 97 are directly related to the size of the parameter $\bar{G}$, which is an upper bound for the vector $G($.$) , which$ consists of elements that are sine and cosine functions of the attitude-tracking errors. These elements were introduced in Equation 69 to 71. In the above development, a conservative approach is taken about this upper bound by simply stating that since these vector elements are sine and cosine functions of the attitude errors, the vector norm has to be bounded by some constant $\bar{G}$. Below, in the second and third parts of the proof, the vector $G($.$) is investigated further to show that$ (1) $\lim _{t \rightarrow \infty} G()=.\underline{0}$, (2) the supremum norm of the vector $G($.) can be made arbitrarily small by choosing the nonlinear adaptive controller constant $\Lambda_{s}$ large enough. In practice, this choice of $\Lambda_{s}$ is limited by the high-frequency unmodeled dynamics of the system together with measurement noise. ${ }^{47}$

From the first part of Theorem 1, which is proven above, it is known that the states $X, Y, Z$, and the adaptive parameter errors $\tilde{K}_{x}, \tilde{K}_{r}$, and $\tilde{D}$ are bounded. Therefore, for a bounded reference $r$, the control input (Equation 35) is bounded. This implies that the total thrust $u_{1}$ determined in Equation 50 is bounded, which shows that the desired attitude angles $\Phi_{d}$ and $\Theta_{d}$, calculated in Equations 51 and 52, which are passed to the inner loop controller as reference inputs, are also bounded. Therefore, the attitude control loop, explained in subsection 3.3 can be shown to be asymptotically stable, ${ }^{47}$ meaning that the attitude-tracking error $\tilde{\alpha}_{\omega}=\left[\begin{array}{lll}\left(\Phi_{d}-\Phi\right) & \left(\Theta_{d}-\Theta\right) & \left(\Psi_{d}-\Psi\right)\end{array}\right]^{T}$ converges to zero asymptotically. Once this is established, it is straightforward to see that $\lim _{t \rightarrow \infty} G(t)=\underline{0} \in \mathfrak{R}^{3}$. This completes the proof of the second part of Theorem 1.
It can be shown that the derivative of the following Lyapunov function,

$$
V_{1}(t)=\frac{1}{2}\left[s^{T} M s+\tilde{I}_{U A V} \Gamma_{I}^{-1} \tilde{I}_{U A V}\right],
$$

is negative semidefinite. ${ }^{47}$ This implies that

$$
\begin{aligned}
& \frac{1}{2}\left[s(t)^{T} M(t) s(t)+\tilde{I}_{U A V}(t) \Gamma_{I}^{-1} \tilde{I}_{U A V}(t)\right] \\
& \quad \leqslant \frac{1}{2}\left(s(0)^{T} M(0) s(0)+\tilde{I}_{U A V}(0)^{T} \Gamma_{I}^{-1} \tilde{I}_{U A V}(0)\right), \forall t \geqslant 0 .
\end{aligned}
$$

After some manipulation, it is obtained from Equation 100 that

$$
\begin{aligned}
& \frac{1}{2}\left[s(t)^{T} M(t) s(t)+\tilde{I}_{U A V}(t) \Gamma_{I}^{-1} \tilde{I}_{U A V}(t)\right] \\
& \quad \leqslant \frac{1}{2}\left(s(0)^{T} M(0) s(0)+\tilde{I}_{U A V}(0)^{T} \Gamma_{I}^{-1} \tilde{I}_{U A V}(0)\right), \forall t \geqslant 0 .
\end{aligned}
$$

After some manipulation, it is obtained from Equation 100 that

$$
\|s(t)\| \leqslant\left(\left(K-\lambda_{\min }\left(\Gamma_{I}^{-1}\right)\left\|\tilde{I}_{U A V}\right\|^{2}\right) / \bar{\lambda}_{\max }(M)\right)^{1 / 2},
$$

where

$$
K=\frac{1}{2}\left(s(0)^{T} M(0) s(0)+\tilde{I}_{U A V}(0)^{T} \Gamma_{I}^{-1} \tilde{I}_{U A V}(0)\right) .
$$

From the definition of $s$ given in Equation 57, it is obtained that

$$
\left\|\tilde{\alpha}_{\omega}\right\| \leqslant\|s(t)\| \Lambda_{s}^{-1}\left(I-e^{-t \Lambda_{s}}\right) \leqslant\|s(t)\| \Lambda_{s}^{-1} .
$$

Using Lemma 2 and Equations 101 and 103, it is straightforward to prove the third part of Theorem 1 . This completes the proof of the third part of Theorem 1.

Remark 7. The implication of Equation 79 is that the "disturbance" $\left[g_{1}(.) g_{2}(.) g_{3}(.)\right]^{T}$ introduced to the closed-loop system (Equation 82), because of the dynamics of the attitude control loop, converges to zero. The speed of convergence is determined by the selection of the nonlinear adaptive control parameters. For all practical purposes, the convergence of the disturbance to zero means that the origin remains to be an equilibrium point of the proposed closed-loop control system structure even though robustfiying terms are used in the adaptive control laws (Equations 36-38).

Remark 8. The implication of Equation 80 is that the sufficiency condition (Equation 97) is not too restrictive since $\bar{G}$ can be made small with a large enough $\Lambda_{s}$. As mentioned earlier, the size of $\Lambda_{s}$ is limited by measurement noise and unmodeled high frequency dynamics.

\section{4 | IMPLEMENTATION SCENARIO}

To examine the behavior of the tilt-wing UAV, during takeoff (quadrotor mode), horizontal flight (fixed wing mode), and transitions between these modes, we created a flight scenario as shown in Figure 4. The UAV takes off 
vertically with $90^{\circ}$ wing angles (0-10 seconds), and after reaching a desired altitude, it changes its wing angles to $20^{\circ}$ (10-20 seconds). After flying in horizontal mode for about $650 \mathrm{~m}$ (20-65 seconds), it changes its wing angles back to $90^{\circ}$, while slowing down (65-100 seconds). Then, it lands as a quadrotor (100-110 seconds). It is noted that during level flight, at $t=61$ seconds, 2 batteries, wing covers and winglets, fall, all from the right wing. Furthermore, Dryden wind gust model ${ }^{48}$ is used to create external disturbances. Below, the changes in mass, moment of inertia, and center of gravity are investigated because of wing movements and failures. Also, a trajectory generation method is given.

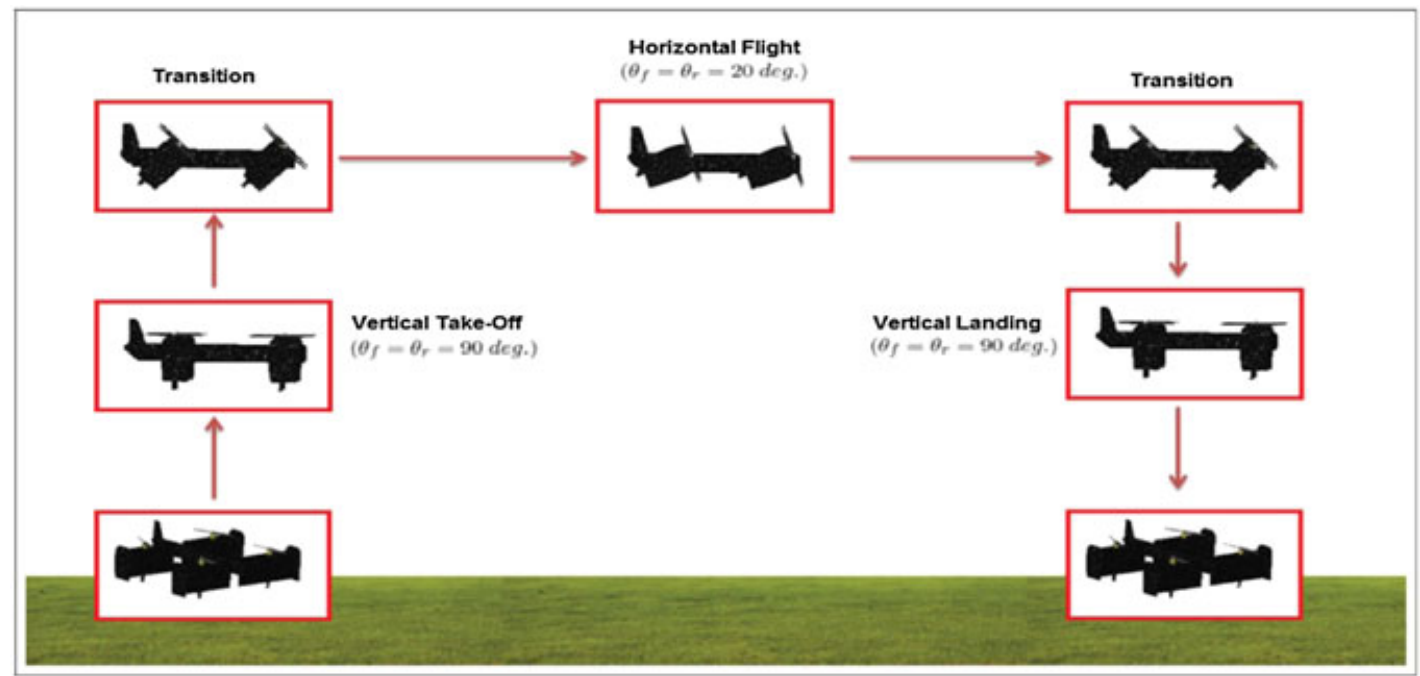

FIGURE 4 Flight scenario. [Colour figure can be viewed at wileyonlinelibrary.com]
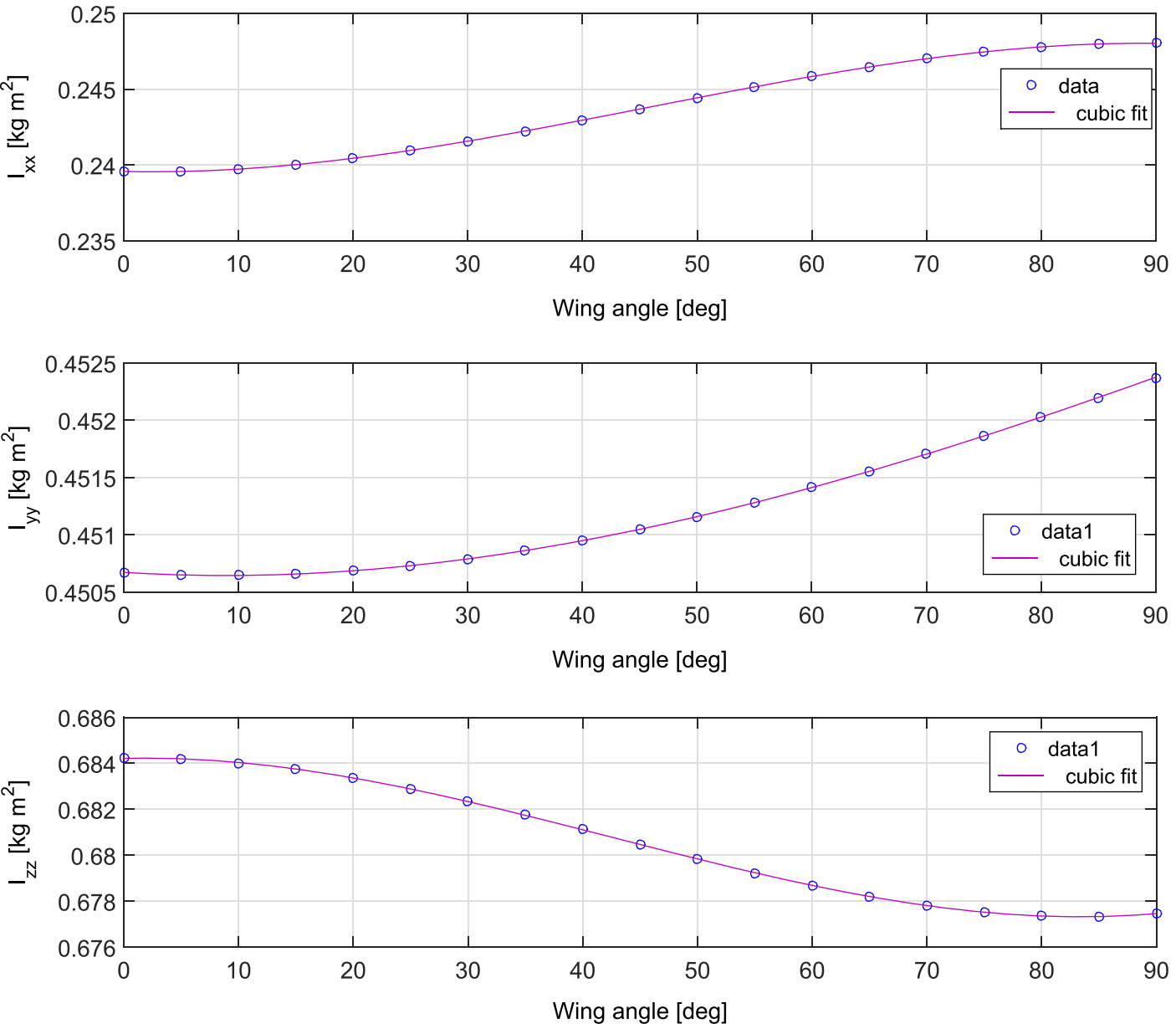

FIGURE 5 Inertia variations during transition from quadrotor mode to fixed-wing mode, before the failure. [Colour figure can be viewed at wileyonlinelibrary.com] 
Remark 9. The above trajectory description also explains the proposed method for the transitions between quadrotor and fixed-wing modes: After a vertical takeoff with $90^{\circ}$ wing angles, the first transition occurs from the quadrotor mode to fixed-wing mode, where the altitude is kept fixed and wing angles are moved from $90^{\circ}$ to $20^{\circ}$. During this transition, the aircraft pitch angle increases to prevent the loss of the lift force. After the wings obtain $20^{\circ}$ angle, the aircraft moves forward horizontally as a fixed-wing aircraft causing the pitch angle to go back to $0^{\circ}$. As the aircraft gets closer to the target destination, wings start to move from $20^{\circ}$ angle to $90^{\circ}$ and the aircraft starts to slow down until it comes to a full stop in the air while keeping the altitude constant. When the aircraft stops its horizontal movement, the wing angles reach $90^{\circ}$ and then the aircraft, now in the quadrotor mode, lands on the ground vertically. The pitch and wing-angle variations during these transitions can be seen more clearly in the simulation results in Section 5.

\section{1 | Moment of inertia variations during transition stages}

The vehicle's computer-aided design model generated in SolidWorks was used to extract the principal moment of inertia changes during the transition. For the transition from quadrotor mode to fixed-wing mode, wing angles were changed from $90^{\circ}$ to $0^{\circ}$ with $5^{\circ}$ intervals, and for each interval, principal moment of inertias was calculated in SolidWorks. Then, cubic polynomials are used for curve fitting. The resulting curves can be seen in Figure 5 .

The same procedure is used to calculate the variations in the moment of inertias during the transition from the fixed-wing mode to quadrotor mode. However, during this transition, the UAV model is different than the one in the first transition because of the missing parts that are lost at the moment of failure at $t=61$ seconds. The resulting curves are presented in Figure 6.

In the simulations, these curves were used to obtain the parameter changes during the transition stages and during the failure. Percent changes in these system parameters are presented in Table 1.

\section{2 | Center of gravity variation due to the failure}

In addition to moment of inertia and mass changes, center of gravity of UAV changes with the failure. This change is modeled as an external disturbance to UAV position dynamics, which consists of the moments $M_{x}, M_{y}$, and $M_{z}$ that are calculated as
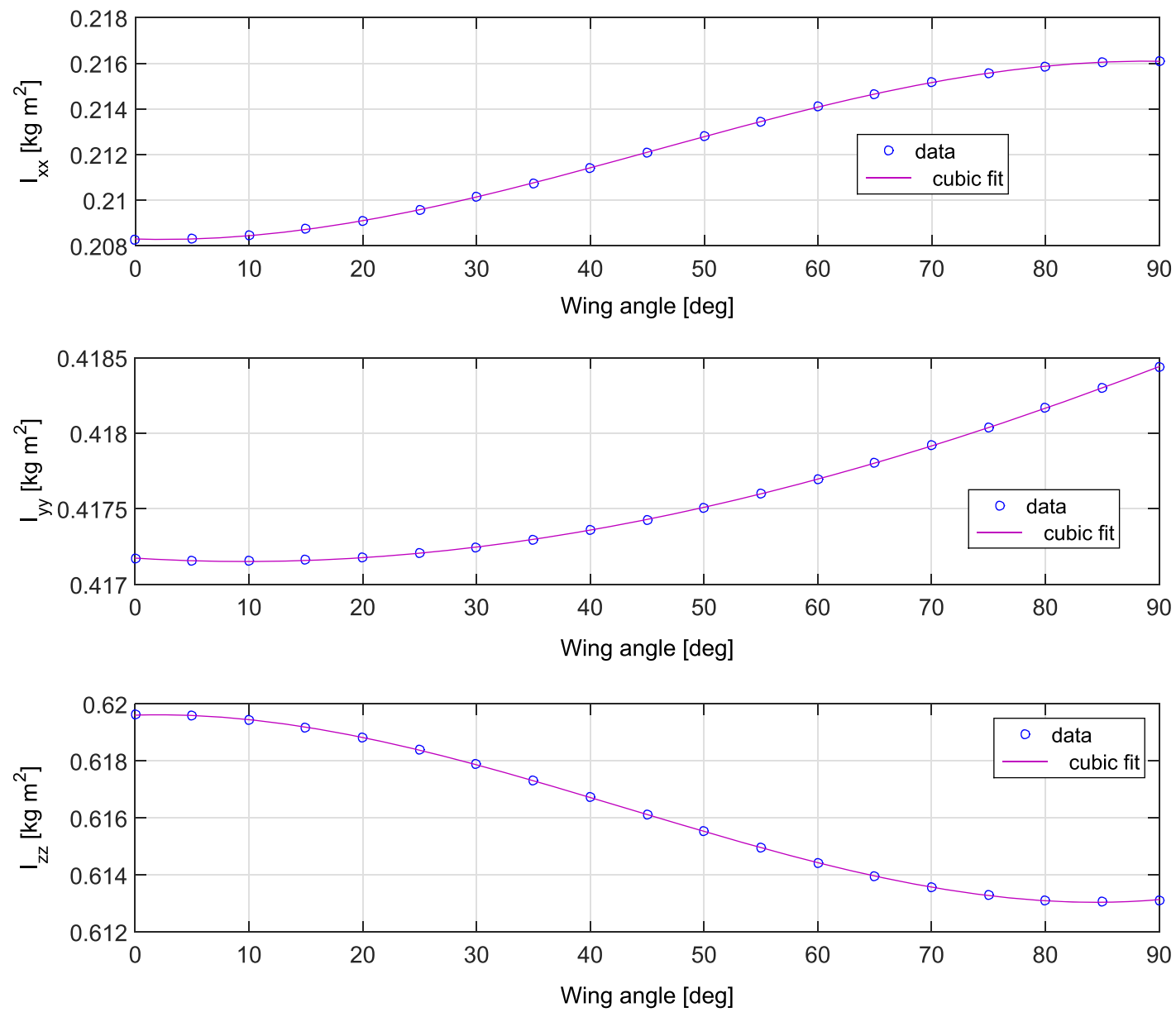

FIGURE 6 Inertia variations during transition from fixed-wing mode to quadrotor mode, after the failure. [Colour figure can be viewed at wileyonlinelibrary. com] 
TABLE 1 Percent changes of principal moment of inertias and mass

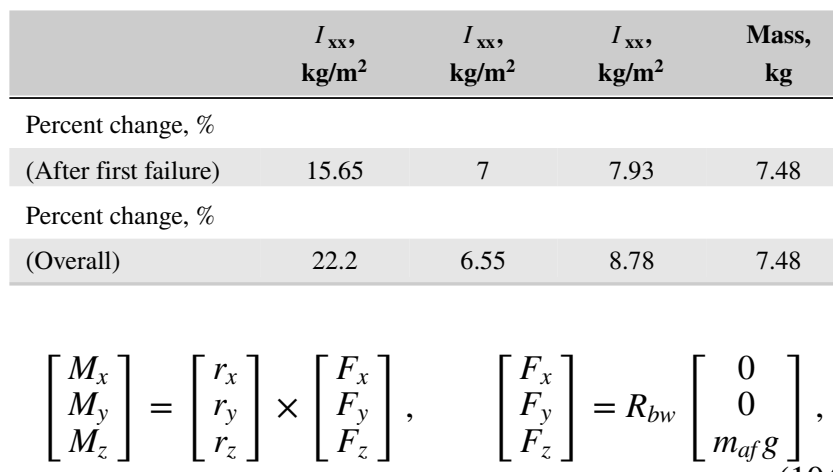

where $R_{b w}$ is the rotation matrix that gives orientation of the body frame with respect to the world frame, $g$ is the gravitational accelaration, $m_{a f}$ is the mass of the UAV after the failure, and $r_{x}, r_{y}$, and $r_{z}$ are distances of the center of gravity to the original position before the failure, measured along the axes.

\section{3 | Trajectory generation}

\subsection{1 | Forward velocity determination}

Tilt-wing UAV tilts its wings during long-duration flights to benefit from the lift forces and flies like a fixed-wing UAV as presented in Figure 1, right picture. However, position reference along the $\mathrm{x}$-axis of world coordinate frame may force the vehicle to fly with relatively slow velocities, which results in a dramatic increase at the pitch angle. Therefore, a minimum forward velocity should be identified that results in a reduced pitch angle during horizontal flight. In this study, a zero-degree pitch angle is targeted.

To obtain the minimum forward velocity that will lead to a zero-degree pitch angle during horizontal flight, we recalled the aerial vehicle position dynamics along the z-axis:

$$
\ddot{Z}=\frac{1}{m}\left[\left(-s_{\theta} c_{\theta_{f}}-c_{\varphi} c_{\theta} s_{\theta_{f}}\right) u_{1}+m g+W_{z}\right],
$$

where sine and cosine of the angles are denoted by $s$ and $c$, respectively. There should be a zero net force along the z-axis (ie, $m \ddot{Z}=0$ ) for a level flight. Additionally, pitch angle should be set to zero, which results in

$$
c_{\varphi} s_{\theta_{f}} u_{1}-m g=W_{z} .
$$

Aerodynamic forces along $\mathrm{x}$-axis, $\mathrm{y}$-axis, and $\mathrm{z}$-axis of the world coordinate frame can be defined as

$$
\begin{aligned}
W(\zeta) & =\left[W_{x}, W_{y}, W_{z}\right]^{T} \\
& =\left[R_{w b}\left[\begin{array}{c}
2\left(\left(F_{D}^{f}\left(\theta_{f}, v_{x}, v_{z}\right)+F_{D}^{r}\left(\theta_{r}, v_{x}, v_{z}\right)\right)\right. \\
0 \\
2\left(\left(F_{L}^{f}\left(\theta_{f}, v_{x}, v_{z}\right)+F_{L}^{r}\left(\theta_{r}, v_{x}, v_{z}\right)\right)\right.
\end{array}\right]\right],
\end{aligned}
$$

where $R_{w b}$ is the rotation matrix between world and body frame and $F_{L}^{i}\left(\theta_{f}, v_{x}, v_{z}\right)$ and $F_{D}^{i}\left(\theta_{f}, v_{x}, v_{z}\right)$ are the lift and drag forces produced by the wings $(i=f, r$ subscripts denote front and rear angles, respectively). To simplify the analysis, front and rear wing angles assumed to be equal $\left(\theta_{f}=\theta_{r}\right)$. Therefore, lift and drag forces are defined as

$$
\begin{aligned}
& F_{L}^{f}\left(\theta_{f}, v_{x}, v_{z}\right)=F_{L}^{r}\left(\theta_{r}, v_{x}, v_{z}\right)=F_{L}, \\
& F_{D}^{f}\left(\theta_{f}, v_{x}, v_{z}\right)=F_{D}^{r}\left(\theta_{r}, v_{x}, v_{z}\right)=F_{D} .
\end{aligned}
$$

From Equation 106, wing forces along z-axis becomes

$$
W_{z}=-s_{\theta}\left(4 F_{D}\right)+c_{\varphi} c_{\theta}\left(4 F_{L}\right) .
$$

If the pitch angle is zero, then $W_{z}$ becomes

$$
W_{z}=c_{\varphi}\left(4 F_{L}\right) .
$$

Substituting $W_{z}$ in Equation 105, the lift force that is necessary for a level flight can be found as

$$
F_{L}=\frac{c_{\varphi} s_{\theta_{f}} u_{1}-m g}{4 c_{\varphi}}
$$

The lift and drag forces are given as

$$
\left[\begin{array}{c}
F_{D}^{i} \\
0 \\
F_{L}^{i}
\end{array}\right]=R\left(\theta_{i}-\alpha_{i}\right)\left[\begin{array}{c}
\frac{1}{2} C_{D}\left(\alpha_{i}\right) \rho A v_{\alpha}^{2} \\
0 \\
\frac{1}{2} C_{L}\left(\alpha_{i}\right) \rho A v_{\alpha}^{2}
\end{array}\right],
$$

where $\rho$ is the air density, $A$ is the wing planform area, and $R\left(\theta_{i}-\alpha_{i}\right)$ is the rotation matrix for the rotation around $\mathrm{y}$-axis that decomposes the forces on the wings onto the body axes. Defining $\beta=\theta_{i}-\alpha_{i}, R(\beta)$ becomes

$$
R(\beta)=\left[\begin{array}{ccc}
c_{\beta} & 0 & s_{\beta} \\
0 & 1 & 0 \\
-s_{\beta} & 0 & c_{\beta}
\end{array}\right] .
$$

$v_{\alpha}$ is the airstream velocity, which is defined as

$$
v_{\alpha}=\sqrt{v_{x}^{2}+v_{z}^{2}},
$$

where $v_{x}$ and $v_{z}$ are UAV's velocities along $\mathrm{x}$-axis and $\mathrm{y}$-axis of the body coordinate frame. $\alpha_{i}$ is the effective angle of attack, which is defined as $\alpha_{i}=\theta_{i}-\left(-\operatorname{atan}\left(v_{z}, v_{x}\right)\right)$. Using Equations 107 and 108, it is obtained that

$$
-2 s_{\beta} C_{D} \rho A v_{\alpha}^{2}+2 c_{\beta} C_{L} \rho A v_{\alpha}^{2}=\frac{c_{\varphi} s_{\theta_{f}} u_{1}-m g}{c_{\varphi}} .
$$

The minimum forward velocity in the body coordinate frame that can achieve zero-degree pitch angle is obtained using Equations 109 and 110 as

$$
v_{x}=\sqrt{\frac{c_{\varphi} s_{\theta_{f}} u_{1}-m g}{2 c_{\beta} c_{\varphi} C_{L} \rho A-2 s_{\beta} c_{\varphi} C_{D} \rho A}-v_{z}^{2}} .
$$

Using the transformation of linear velocities between the body and the world frames, $V_{w}=R_{b w} V_{b}$, minimum forward linear velocity in the world frame that can achieve zero-degree pitch angle can be identified as

$$
\dot{X}=c_{\psi} c_{\theta} v_{x}+s_{\varphi} s_{\theta} c_{\psi} v_{y}-c_{\varphi} s_{\psi} v_{y}+c_{\varphi} s_{\theta} c_{\psi} v_{z}+s_{\varphi} s_{\psi} v_{z}
$$





FIGURE $7 \quad X$ tracking. [Colour figure can be viewed at wileyonlinelibrary.com]
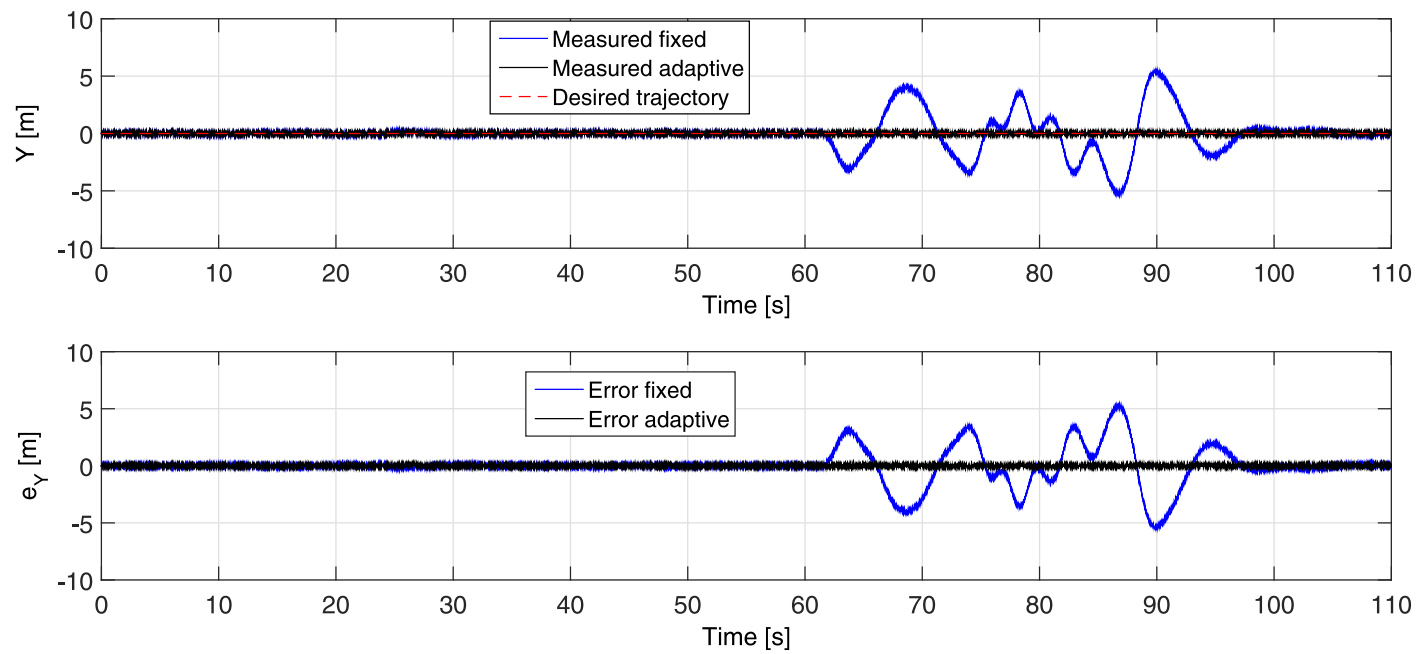

FIGURE 8 Y tracking. [Colour figure can be viewed at wileyonlinelibrary.com]
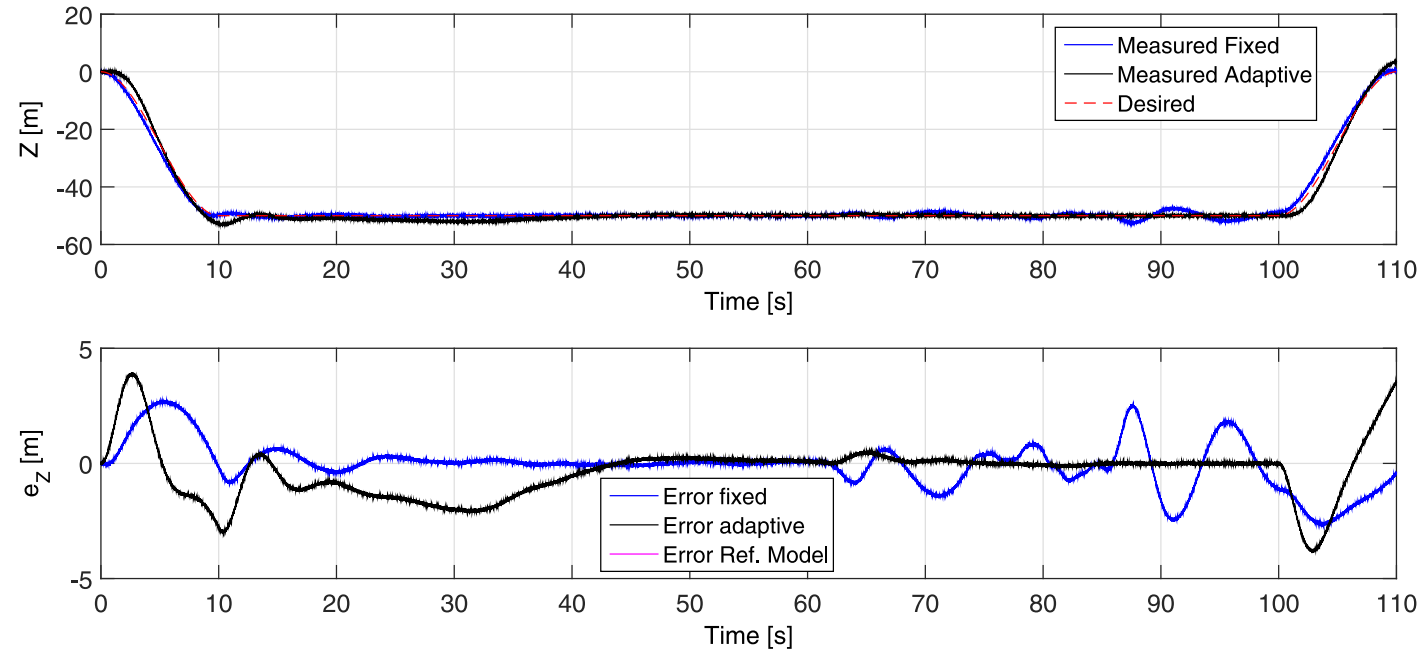

FIGURE 9 Z tracking. [Colour figure can be viewed at wileyonlinelibrary.com] 

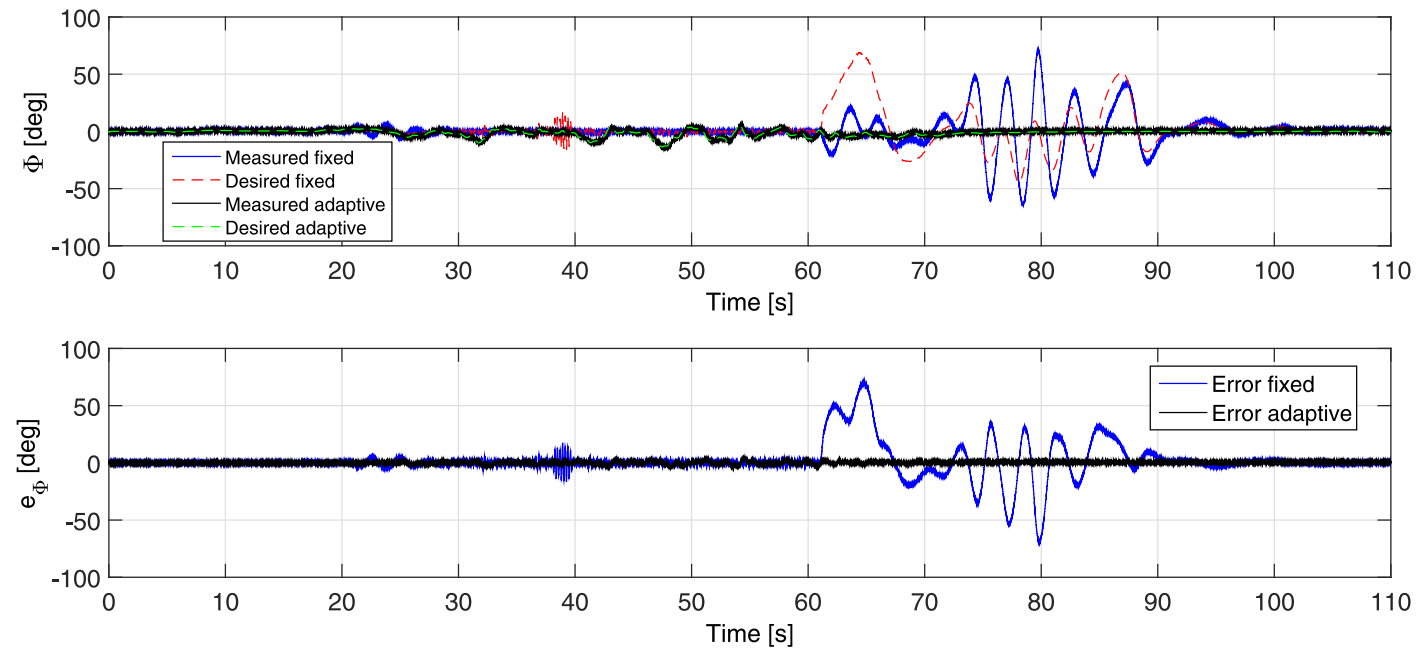

FIGURE $10 \Phi$ tracking. [Colour figure can be viewed at wileyonlinelibrary.com]
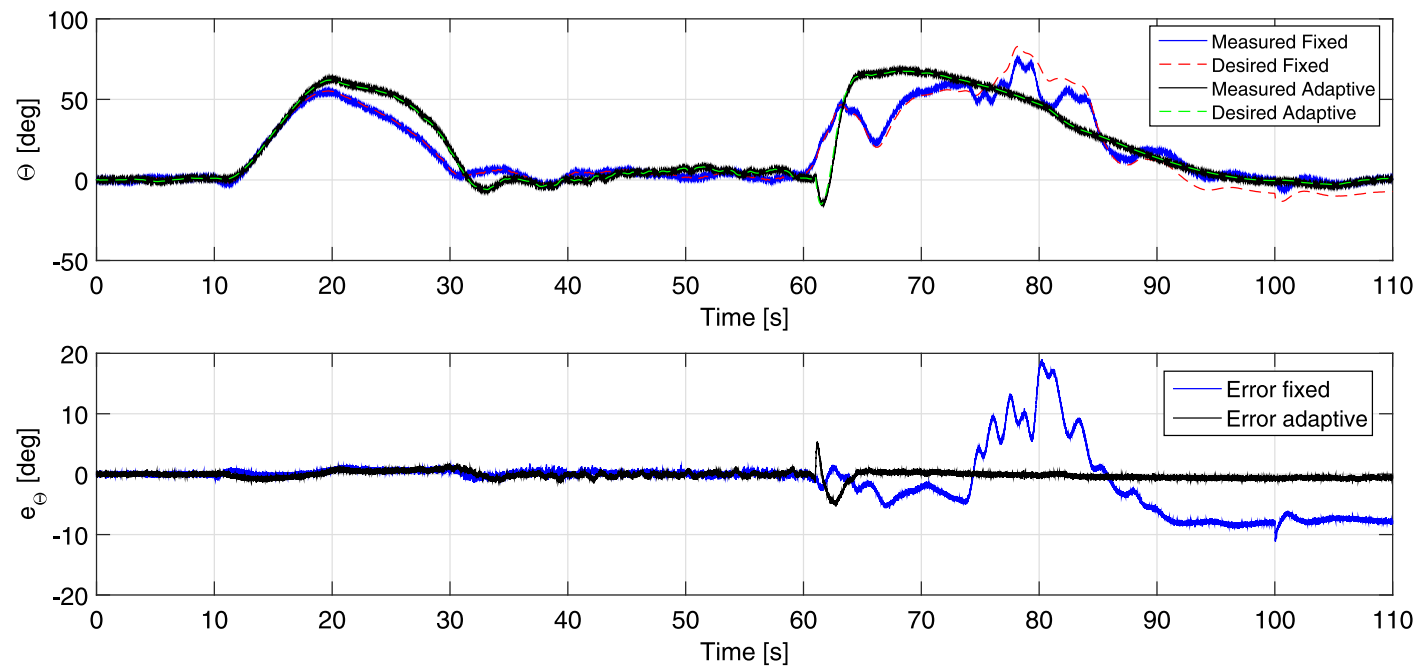

FIGURE $11 \Theta$ tracking. [Colour figure can be viewed at wileyonlinelibrary.com]
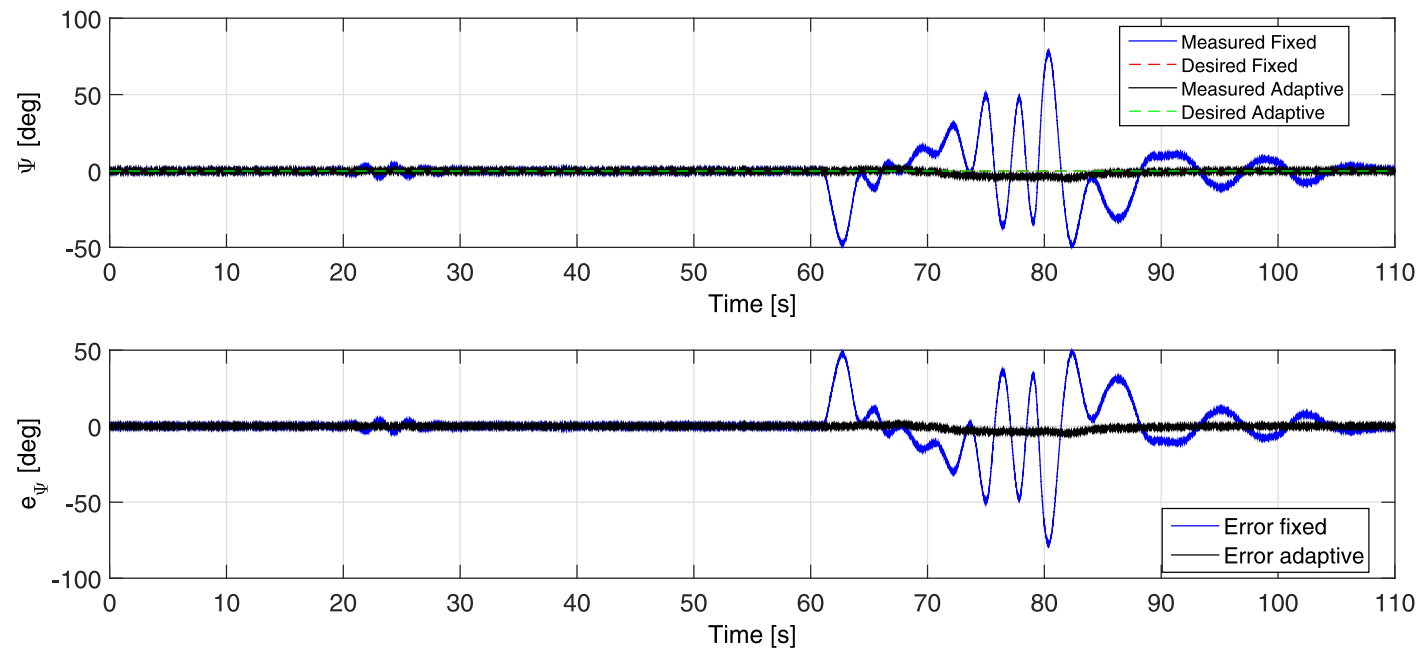

FIGURE $12 \Psi$ tracking. [Colour figure can be viewed at wileyonlinelibrary.com] 


\subsection{2 | Velocity profile determination}

If minimum velocity, which creates enough lift forces to sustain level flight of the UAV, is not achieved, UAV starts to increase its pitch angle. Therefore, a suitable trajectory is generated along the $\mathrm{x}$-axis by using so-called linear segments with parabolic blends. This trajectory has a trapezoidal velocity profile where a constant velocity can be imposed between predefined time instances, as well as linear (ramp) velocity variations. The resulting trajectory consists of quadratic and linear polynomials with smooth blending between them. A more detailed analysis of linear segments with parabolic blends can be found in 1 study. ${ }^{49}$

Remark 10. In this study, we chose to provide a practical approach for trajectory generation, where a horizontal flight with minimum pitch angle is required. However, there may be applications where the goal is minimizing energy consumption and the resultant pitch angle and speed and wing angle can be tolerated. In that case, the necessary wing angle and forward speed combination that would result in maximum lift to drag ratio should be calculated and a suitable trajectory should be generated to achieve this combination.

\section{5 | SIMULATION RESULTS}

In this section, the performance of the proposed adaptive control framework is investigated using the scenario explained in the previous section. It is noted that all the simulations are conducted using a high-fidelity simulation model, in the presence of uncertainties, disturbances, and measurement noise. In addition to the changes in the mass, moment of inertia, and center of gravity, due to the failure, a $10 \%$ uncertainty is assumed in the actuator powers. Also, a 20\% actuator power loss is assumed because of the failure at $t=61$ seconds where 2 batteries fall. The performance of the fixed controller used in earlier studies, ${ }^{37}$ which is a cascade of a PID controller (outer loop) and a feedback linearazition + PID controller (inner loop), is also given as a comparison. As expected, the
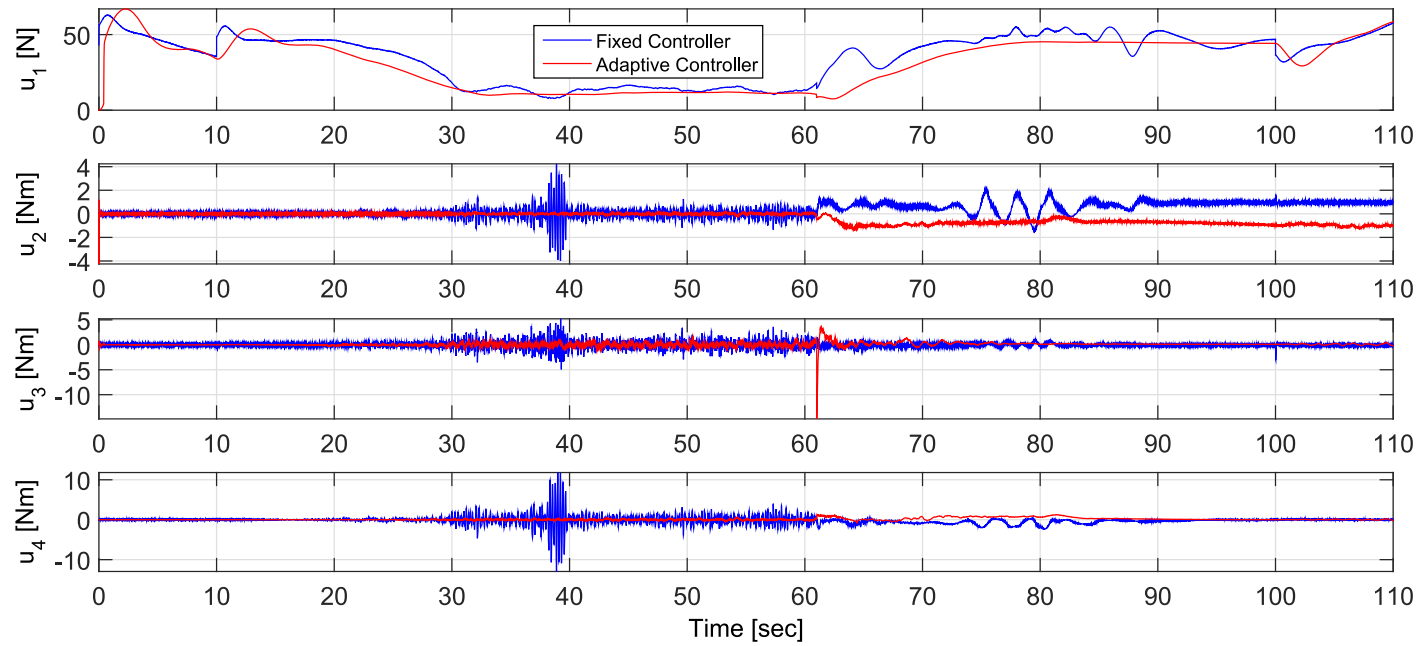

FIGURE 13 Control inputs. [Colour figure can be viewed at wileyonlinelibrary.com]
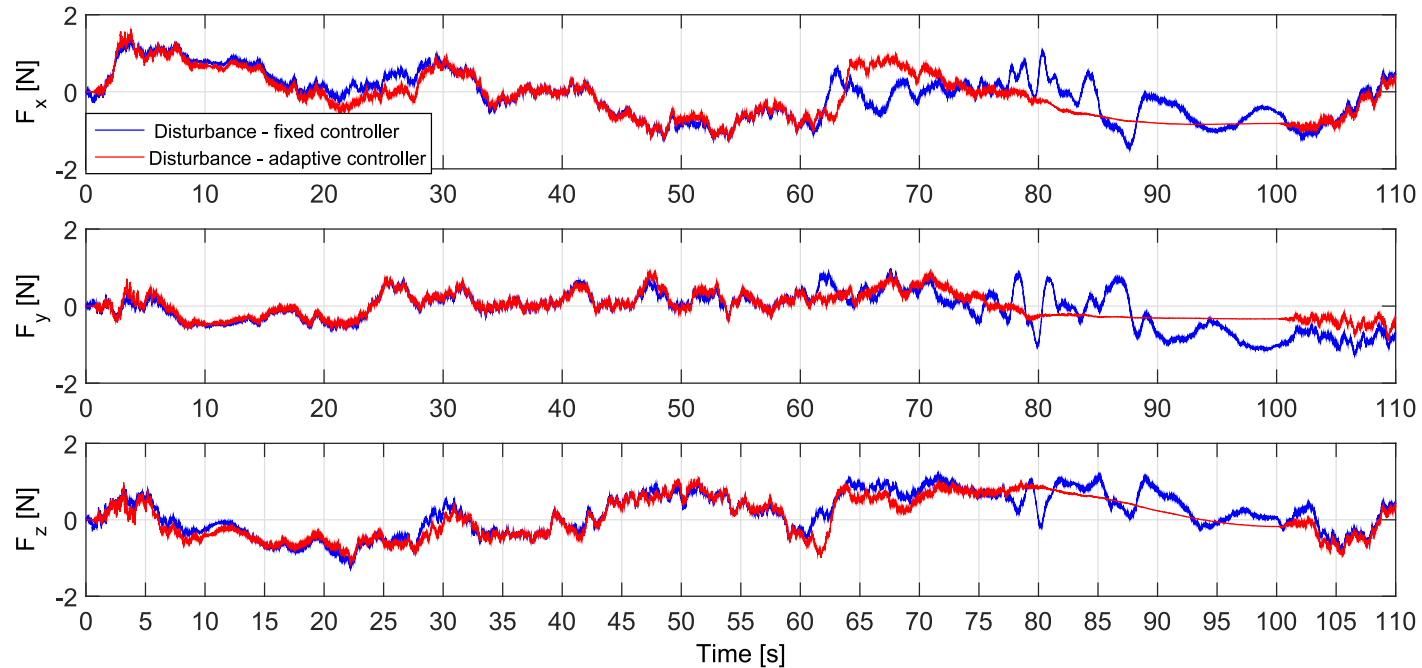

FIGURE 14 Wind disturbances. [Colour figure can be viewed at wileyonlinelibrary.com] 




FIGURE 15 Wing angles. [Colour figure can be viewed at wileyonlinelibrary.com]
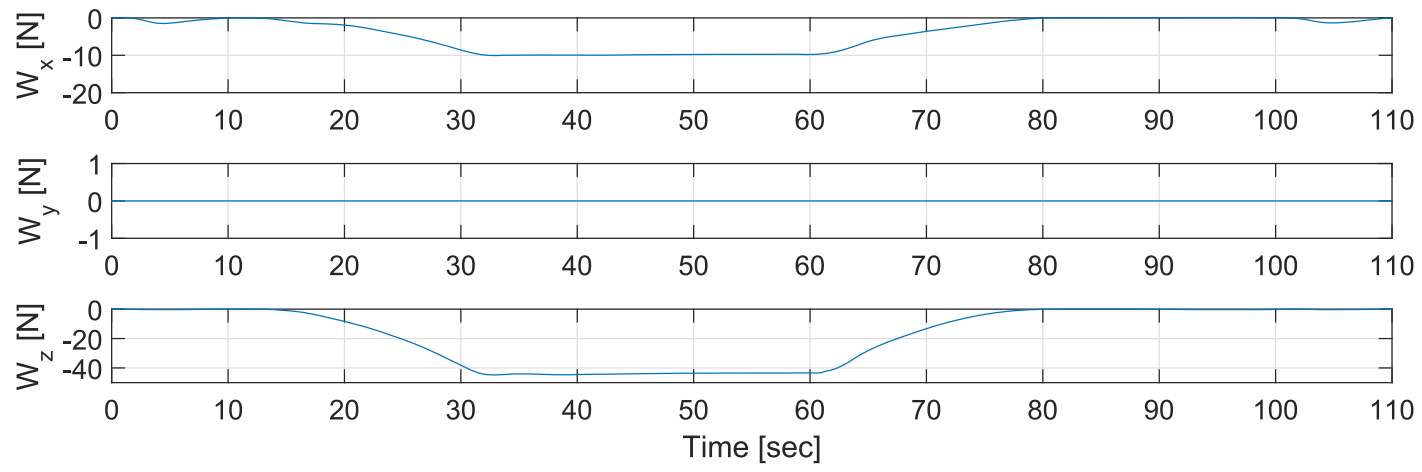

FIGURE 16 Wing forces. [Colour figure can be viewed at wileyonlinelibrary.com]

adaptive controller outperforms the fixed controller due to its adaptability to uncertainties.

Figures 7 to 9 show the performance of the controllers for the position trajectory tracking. The closed-loop system with the adaptive controller deviates much less from the trajectory compared with the fixed controller, especially after the failure instant at $t=61$ seconds.

Figures 10 to 12 show the attitude-tracking curves. As seen from the figures, the system with the proposed controller can keep its roll $(\Phi)$ and yaw $(\Psi)$ angles close to zero. On the other hand, UAV shows large roll and yaw-angle variations when the fixed controller is in charge. The 2 controllers are comparable in the variations in the pitch angle $(\Theta)$ until the failure instant, but after the failure at $t=61$ seconds, the pitch angle varies more smoothly with the proposed controller compared with the fixed controller.

It is noted that although its performance is not as good as the adaptive controller, the fixed controller can still keep the closed-loop system under the influence of parametric uncertainties, center of gravity, mass and moment of inertia changes as well as actuator power losses, wind disturbances, and measurement noise. However, the fixed controller pays the price by outputting very noisy control inputs. As seen from Figure 13, especially between $t=30-60$ seconds, the fixed controller output have high-amplitude high-frequency components. It is noted that at this time interval, the UAV's forward speed reaches its maximum value of $\approx 50 \mathrm{~km} / \mathrm{h}$. On the other hand, the adaptive control inputs are smoother. The wind disturbances can be seen in Figure 14, where the disturbances acting on the UAV are seen to be different at certain time intervals for different controller implementations due to speed, orientation, and altitude differences.

Figure 15 shows the movement of the wings during the flight, and Figure 16 shows the resulting aerodynamic forces acting on the wings. It is seen that a considerable amount of lift is generated together with some drag force. For the energy gain compared to a similar quadrotor without wings to be calculated, it is assumed that the wingless quadrotor would need less force in the $\mathrm{x}$ direction due to zero drag (from the wings) and more force in the $\mathrm{z}$ direction due to the lack of lift. With these considerations, it is calculated that the quad tilt-wing UAV spends $\approx 33 \%$ less energy, compared with a conventional wingless quadrotor, for the flight scenario used in this study.

\section{6 | CONCLUSIONS}

A nonlinear adaptive control framework that works in a hierarchical structure is proposed for the control of a quad tilt-wing UAV. The controller development does not need any linearization of the UAV dynamics. Rigorous stability analysis of the controller is provided. The controller is implemented using a nonlinear, high-fidelity model of the tilt-wing $\mathrm{UAV}$, in the presence of uncertainties, actuator failures, structural failures, center of gravity changes due to these failures, and the effect of wing-angle variations on moment of inertia. The implementation results show that the proposed controller works as intended and performs dramatically better than the fixed controller used in earlier flight tests. 


\section{REFERENCES}

1. Snyder D. The quad tiltrotor: its beginning and evolution. Proc. 56th Annual Forum, American Helicopter Society, Virginia Beach, Virginia, May 2000; 48-61.

2. Lee J, Min B, Kim E. Autopilot design of tilt-rotor UAV using particle swarm optimization method. Proc. International Conference on Control, Automation and Systems, Seoul, Korea, October 2007; 1629-1633.

3. Kendoul F, Fantoni I, Lozano R. Modeling and control of a small autonomous aircraft having two tilting rotors. Proc. 44th IEEE Conference on Decision and Control, and the European Control Conference, Seville, Spain, December 2005; 1297-1302.

4. Dickeson J, Miles D, Cifdaloz O, Wells VL, Rodriguez A. Robust lpv h-inf gain-scheduled hover-to-cruise conversion for a tilt-wing rotorcraft in the presence of cg variations. Proc. 46th IEEE Conference on Decision and Control, New Orleans, LA, December 2007; 2773-2778.

5. Muraoka K, Okada N, Kubo D. Quad tilt wing VTOL UAV: aerodynamic characteristics and prototype flight test. AIAA Infotech@ Aerospace Conference, Seattle, Washington, April 2009; Paper No. AIAA 2009-1834.

6. Suzuki S, Zhijia R, Horita Y, Nonami K, Kimura G, Bando T, Hirabayashi D, Furuya M, Yasuda K. Attitude control of quad rotors QTW-UAV with tilt wing mechanism. J Syst Design Dyn. 2010;4:416-428.

7. Dydek ZT, Annaswamy AA, Lavretsky E. Adaptive control of quadrotor UAVs: a design trade study with flight evaluations. IEEE Trans Control Syst Tecnol. 2013;21(4):1400-1406.

8. Kim K-S, Lee K-J, Kim Y. Reconfigurable flight control system design using direct adaptive method. J Guidance Control Dyn. 2003;26(4):543-550.

9. Bouabdallah S, Noth A, Siegwart R. PID vs LQ control techniques applied to an indoor micro quadrotor. Proc 2004 IEEE/RSJ Int Conference on Intelligent Robots and Systems, Sendai, Japan, September 2004; 2451-2456.

10. Bouabdallah S, Murrieri P, Siegwart R. Towards autonomous indoor micro VTOL. Autonomous Robots. 2005;18(2):171-183.

11. Hoffmann GM, Huang H, Waslander SL, Tomlin CJ. Quadrotor helicopter flight dynamics and control: theory and experiment. AIAA Guidance Navigation and Control Conference and Exhibit, Hilton Head, South Carolina, August 2007; Paper number AIAA 2007-6461.

12. Tayebi A, McGilvray S. Attitude stabilization of a four-rotor aerial robot. In Proc. 43th IEEE Conference on Decision and Control, vol. 2: Atlantis, Paradise Island, Bahamas, 2004;1216-1221.

13. Shin J, Nonami K, Fujiwara D, Hazawa K. Model-based optimal attitude and positioning control of small-scale unmanned helicopter. Robotica. 2005; 23(1):51-63

14. Cowling ID, Yakimenko OA, Whidborne JF, Cooke AK. A prototype of an autonomous controller for a quadrotor uav. European Control Conference, Kos, Greece, 2007;1-8.

15. How JP, Bethke B, Frank A, Dale D, Vian J. Real-time indoor autonomous vehicle test environment. IEEE Control Syst Mag. 2008;28(2):51-64.

16. Benallegue A, Mokhtari A, Fridman L. High-order sliding-mode observer for a quadrotor UAV. Int J Robust Nonlinear Control. 2008; 18(4): $427-440$.

17. Civita ML, Papageorgiou G, Messner WC, Kanade T. Design and flight testing of an h- $\infty$ controller for a robotic helicopter. J Guidance Control Dyn. 2006;29(2):485-494.

18. Koo TJ, Sastry S. Output tracking control design of a helicopter model based on approximate linearization. Proc. Conference on Decision and Control, Tampa, Florida, USA, 1998;3635-3640.

19. Kim HJ, Shim DH. A flight control system for aerial robots: algorithms and experiments. Control Eng Pract. 2003;11(12):1389-1400.

20. Reiner J, Balas GJ, Garrard WL. Robust dynamic inversion for control of highly maneuverable aircraft. J Guidance Control Dyn. 1995; 18(1): $18-24$.

21. Castillo P, Dzul A, Lozano R. Real-time stabilization and tracking of a four-rotor mini rotorcraft. IEEE Transactions on Control Systems Technology. 2004;12(4):510-516.

22. Frazzoli E, Dahleh MA, Feron E. Trajectory tracking control design for autonomous helicopters using a backstepping algorithm. Proc. Amer. Control Conf., Chicago, Illinois, USA, June 2000;4102-4107.
23. Mahony R, Hamel T. Robust trajectory tracking for a scale model autonomous helicopter. Int $J$ Robust Nonlinear Control. 2004; 14(12):1035-1059.

24. Bouabdallah S, Siegwart R. Backstepping and sliding-mode techniques applied to an indoor micro quadrotor. Proceedings of IEEE International Conference on Robotics and Automation, Barcelona, Spain, 2005; 2247-2252.

25. Hua M, Hamel T, Morin P, Samson C. A control approach for thrust-propelled underactuated vehicles and its application to VTOL drones. IEEE Trans Autom Control. 2009;54(8):1837-1853.

26. Pflimlin JM, Hamel T, Soueres P, Mahony R. A hierarchical control strategy for the autonomous navigation of a ducted fan flying robot. Proceedings of IEEE International Conference on Robotics and Automation, Orlando, Florida, 2006;2491-2496.

27. Esteban S, Gordillo F, Aracil J. Lyapunov-based stability analysis of a three-time scale model for a helicopter on a platform. Proceedings of IFAC Symposium on Automatic Control in Aerospace, Tolosana, France, 2007;467-472.

28. Das A, Subbarao K, Lewis F Dynamic inversion with zero-dynamics stabilisation for quadrotor control. IET Control Theor Appl. 2009;3(3):303-314.

29. Bertrand S, Guenard N, Hamel T, Piet-Lahanier H, Eck L. A hierarchical controller for miniature VTOL UAVs: Design and stability analysis using singular perturbation theory. Control Eng Pract. 2011;19(10):1099-1108.

30. Liu H, Xi J, Zhong Y. Robust motion control of quadrotors. Journal of the Franklin Institute. 2014;12:5494-5510.

31. Kendoul F. Survey of advances in guidance, navigation, and control of unmanned rotorcraft systems. J Field Rob. 2012;29(2):315-378.

32. Johnson EN, Kannan SK. Adaptive trajectory control for autonomous helicopters. J Guidance Control Dyn. 2005;28(3):524-538.

33. Lee D, Kim HJ, Sastry S. Feedback linearization vs. adaptive sliding mode control for a quadrotor helicopter. International Journal of Control, Automation and Systems. 2009;7(3):419-428.

34. Nicol C, Macnab CJB, Ramirez-Serrano A. Robust adaptive control of a quadrotor helicopter. Mechatronics. 2011;21(6):927-938.

35. Palunko I, Fierro R. Adaptive control of a quadrotor with dynamic changes in the center of gravity. Proceedings of 18th IFAC World Congress, Milano, Italy, 2011;2626-2631.

36. Nonami K, Kendoul F, Suzuki S, Wang W, Nakazawa D. Chapter: Mathematical Modeling and Nonlinear Control of VTOL Aerial Vehicles. In Autonomous Flying Robots. Springer: Japan, 2010.

37. Cetinsoy E, Dikyar S, Hancer C, Oner KT, Sirimoglu E, Unel M, Aksit MF. Design and construction of a novel quad tilt-wing UAV. Mechatronics. 2012; 22:723-745.

38. Cetinsoy E, Hancer C, Oner KT, Sirimoglu E, Unel M. Aerodynamic design and characterization of a quad tilt-wing UAV via wind tunnel tests. J Aerosp Eng. 2012;25(4):574-587.

39. Hancer C, Oner KT, Sirimoglu E, Cetinsoy E, Unel M. Robust position control of a tilt-wing quadrotor. In Proc. 49th IEEE Conference on Decision and Control, vol. 2: Atlanta, GA, 2010;4908-4913.

40. Hancer C, Oner KT, Sirimoglu E, Cetinsoy E, Unel M. Robust hovering control of a quad tilt-wing UAV. Proc. 36th Annual Conference on IEEE Industrial Electronics Society, Glendale, AZ, November 2010; $1615-1620$.

41. Yildiz Y, Unel M, Demirel AE. Adaptive nonlinear hierarchical control of a quad tilt-wing UAV. Proceedings of European Control Conference, Linz, Austria, 2015; 3623-3628. (http://www.syslab.bilkent.edu.tr/publications/ ECC2015YUD.pdf).

42. Lavretsky E. Adaptive control: Introduction, overview, and applications. In Lecture notes from IEEE Robust and Adaptive Control Workshop, Seattle, Washington, 2008.

43. Narendra KS, Annaswamy AM. Stable Adaptive Systems. Dover Publications: New York, 2005.

44. Slotine J-J. Adaptive robot control: a new perspective. Proc. 26th Conference on Decision and Control, Los Angeles, CA, December 1987.

45. Barton JD. Fundamentals of small unmanned aircraft flight. Johns Hopkins APL Tech Digest. 2012;31(2):132-149. 
46. Kendoul F. Modelling and control of unmanned aerial vehicles, and development of a vision-based autopilot for small rotorcraft navigation. PhD Thesis, 2007.

47. Slotine J-JE, Li W. Applied Nonlinear Control. Prentice-Hall: Eagle Wood Cliffs, NJ, 1991.

48. Waslander SL, Wang C. Wind disturbance estimation and rejection for quadrotor position control.AIAA Infotech@Aerospace Conference and AIAA Unmanned. Unlimited Conference, Seattle, WA, 2009.

49. Spong MW, Vidyasagar M. Robot Dynamics and Control (1st edn.) John Wiley \& Sons, Inc.: New York, NY, USA, 1989.
How to cite this article: Yildiz $Y$, Unel $M$, Demirel AE. Nonlinear hierarchical control of a quad tilt-wing UAV: An adaptive control approach. Int $J$ Adapt Control Signal Process. 2017;31:1245-1264. https://doi.org/10.1002/acs.2759 